\title{
Non-Gaussianities in models of inflation with large and negative entropic masses
}

\author{
Ricardo Z. Ferreira \\ Nordita, KTH Royal Institute of Technology and Stockholm University, \\ Roslagstullsbacken 23, SE-106 91 Stockholm, Sweden \\ E-mail: ricardo.zambujal@su.se
}

\begin{abstract}
Models of inflation where the entropic directions have large and negative masses $\left|m_{s}\right| \gg H$ can be well described by a single-field EFT with an imaginary sound speed $c_{s}$. Among other features, they predict an exponential enhancement of the spectrum of scalar perturbations which however is not inherited by non-Gaussianities. In this work, I complete the calculation of the trispectrum in this EFT by considering the contributions from the contact interaction and the exchange diagram. While for most shapes the trispectrum is approximately constant, I find that for certain configurations where all the momenta collapse to a line the trispectrum is proportional to $\left(m_{s} / H\right)^{5}$ for the contact interaction and to $\left(m_{s} / H\right)^{6}$ for the exchange diagram, as anticipated by previous work. I also discuss the UV sensitivity of the results and argue why the EFT provides a good order of magnitude estimate. In the end, I confront the different predictions for the scalar spectrum against observations. In models where the entropic mass is proportional to a positive power of the slow-roll parameter $\epsilon$, like in hyperinflation, the spectrum grows on small scales and becomes constrained by the overproduction of primordial black holes. Imposing such constraint jointly with the correct amplitude and spectral tilt at CMB scales excludes a large set of potentials. Only those where the spectral tilt is controlled by $m_{s} \delta / H \sim \mathcal{O}(-0.01)$, where $\delta=\dot{\epsilon} /(\epsilon H)$ is the second slow-roll parameter, are likely observationally viable. Finally, the constraints on the bispectrum generically impose $\left|c_{s} m_{s}\right| / H \lesssim 10-20$ while those on the trispectrum give a weaker bound when using the constraints on $g_{\mathrm{NL}}^{\dot{\sigma}^{4}}$ as a proxy. For hyperinflation the bispectrum bound translates into $\omega \lesssim 11$ where $\omega$ is the turning rate in field-space.
\end{abstract}




\section{Contents}

1 Introduction 1

2 Basics of the model $\quad 3$

3 Non-Gaussianities $\quad 4$

3.1 Bispectrum 5

3.1.1 Terms in $\zeta^{\prime 3} \quad 6$

$\begin{array}{lll}3.1 .2 & \text { Terms in } \zeta^{\prime}(\partial \zeta)^{2} & 7\end{array}$

3.2 Trispectrum 8

3.2.1 Quartic interaction 8

$\begin{array}{lr}3.2 .2 \text { Cubic interaction } & 10\end{array}$

4 Observational constraints $\quad 16$

$\begin{array}{lll}4.1 & \text { Power spectrum and spectral index } & 17\end{array}$

$\begin{array}{lll}4.2 & \text { Non-Gaussianities } & 17\end{array}$

5 Conclusion $\quad 20$

$\begin{array}{ll}\text { A Rapid-turn attractors } & 21\end{array}$

B Interaction Hamiltonians $\quad 22$

B.1 Cubic order 22

B.2 Quartic order 23

C Trispectrum: general formulas $\quad 23$

C.1 Contact interaction: contributions from $\mathcal{I}_{3} \quad 24$

C.2 Exchanged diagram: equilateral limits 24

\section{Introduction}

In the inflationary paradigm, what protects the inflaton from large radiative corrections and a new naturalness problem? Symmetries are naturally good mass protectors and have been thoroughly explored, for example, in the case of shift symmetries [1-3]. However, the latest observational data does not seem to point in that direction, at least for the minimal scenarios [4]. Moreover, the symmetry breaking scale generating such Goldstone boson tends to be (super) Planckian thus raising concerns about large gravitational corrections (see [5] for a recent discussion).

Another interesting possibility is to have a dynamical mechanism where multi-field effects counteract the rolling of the inflaton thus effectively allowing for inflation in steeper potentials. In that regard, considerable attention has been given to multi-fields models with a strongly non-geodesic motion (see [6-10] for a few examples). In particular, more recently it has been noted that even when the field space has a negative curvature $R_{\mathrm{fs}}$, of which hyperbolic geometries are prototypical examples [10-21], new slow-roll attractors in the inflationary trajectory are still possible to find. In these attractors, which have been argued to belong all 
to the same class [19], the initially spectator field is typically displaced from its minima and there is a fast turning rate in field-space.

Parallelly to this discussion, it is known that for any two-field system the quadratic action for perturbations assumes a very simple form where the shift symmetric adiabatic curvature perturbation, $\zeta$, couples derivatively to the entropic degree of freedom whose effective mass is controlled by [22]

$$
m_{s}^{2}=V_{; s s}+H^{2}\left(\epsilon R_{\mathrm{fs}} M_{p}^{2}-\eta_{\perp}^{2}\right),
$$

where $V$ is the multi-field potential and $\eta_{\perp}=-V_{, s} /(H \dot{\phi})$ controls the bending of the trajectory in field-space. The covariant derivatives are taken with respect to the field-space metric and the subscript $s$ denotes the entropic direction. As usual, when the entropic direction is massive it can be integrated out yielding a single-field effective field theory (EFT) with a reduced speed of sound [23-25]. However, recently it has been pointed out that even if $m_{s}^{2}$ is large and negative, $-m_{s}^{2} / H^{2} \gg 1$, the entropic direction can still be integrated out using its equation of motion [14]. The resulting theory, valid for wavelengths smaller than $\left|m_{s}\right|$, is still a single-field EFT but with an imaginary sound speed controlled by $c_{s}^{-2}=1+4 \eta_{\perp}^{2} H^{2} / m_{s}^{2}<0$. Remarkably, even if $\eta_{\perp}^{2} \gg 1$ dominates the effective mass $m_{s}$ making it large and negative, the theory is still stable because on superhorizon scales the effective entropic mass is instead $m_{s}^{2}+4 \eta_{\perp}^{2} H^{2}>0$. The consequences though are rather non-trivial. The temporary negative mass causes an exponential growth of $\zeta$ for modes in the window $H \lesssim k / a \lesssim\left|m_{s}\right|$, similarly to what happens in the context of axial couplings to gauge fields during inflation [26-29].

Non-Gaussianities are another feature of the models described by such EFT. Interestingly, they do not inherit the exponential growth but instead a polynomial dependence on $m_{s} / H$. This has been shown for the 3-point function [30] and also for higher-order correlators [31, 32]. Namely, while for most shapes the non-Gaussian parameters are constant in the $m_{s} \gg H$ limit, for certain shapes approaching the collapsing limit, where all momenta in the correlator collapse to a line (see fig. 1), the time integrals become dominated by the UV cutoff with a leading power-law dependence of $\alpha^{3}$ in the bispectrum, and $\alpha^{6}$ in the trispectrum where $\alpha \equiv-\left|c_{s} m_{s}\right|^{2} / H^{2}[30,32]$. The peak on flattened shapes and its UV sensitivity are typical of theories with excited initial states $[33,34]$. It shows that to find a precise result for such shapes one necessarily needs to consider the full multi-field system. However, as I will discuss later on, if the theory quickly approaches that of approximately massless and weakly coupled scalar fields for scales above the cutoff, the UV contribution will be strongly suppressed thus validating the order of magnitude estimation within the EFT.

In this work, I will complete the trispectrum computation by evaluating the contributions from the contact interaction and the exchange diagram (see fig. 2). So far only one term of the exchange diagram has been computed [32]. I find results consistent with what has been found in the literature, i.e., that the exchange diagram is the dominant contribution to the trispectrum and that it peaks on shapes where all the momenta are collinear. Afterwards, I will collect the different predictions of these models for the spectrum of scalar perturbations: amplitude, spectral tilt and non-Gaussianity; and confront them against observations. In some cases, I will make quantitative assessments on the observational viability of the theory while in other the model dependence only allows for qualitative statements.

The paper is organized as follows. In sec. 2 I give the basic ingredients of the EFT with imaginary sound speed. In sec. 3 I look at the non-Gaussianities, first, by revisiting the bispectrum in sec. 3.1 and then by evaluating the trispectrum in sec. 3.2. In sec. 4 I use observations to place constraints on the models. Auxiliary and intermediate expressions are 
presented in the appendix. Namely, app. A briefly discusses 2 rapid-turn attractor models, app. B presents the interaction Hamiltonians and sec. C a few expressions related to the trispectrum including the results for the equilateral shape.

\section{Basics of the model}

The models under discussion are those where the entropic direction ${ }^{1}$ has an effective mass $m_{s}^{2}$ which is large and negative, $-m_{s}^{2} / H^{2} \equiv \alpha^{2} /\left|c_{s}\right|^{2} \gg 1$. In such cases, one can use the singlefield EFT with imaginary sound speed to compute non-Gaussianities [30]. In this section, I review the basic features of the EFT and set the notation for the following sections.

To see why the EFT is a good description let me consider a model with 2 fields $\phi_{1}, \phi_{2}$ described by the Lagrangian

$$
\mathcal{L}=-\frac{1}{2} G_{a b} \partial_{\mu} \phi^{a} \partial^{\mu} \phi^{b}-V,
$$

where $G_{a b}\left(\phi_{1}, \phi_{2}\right)$ is the field-space metric and $V\left(\phi_{1}, \phi_{2}\right)$ the potential energy. Both $G_{a b}$ and $V$ are chosen such that the background is in slow-roll, either the standard slow-roll attractor or a different one. Remarkably, at the level of perturbations, the Lagrangian at quadratic order is generically given by [22]

$$
\mathcal{L}=-\epsilon M_{p}^{2}(\partial \zeta)^{2}-\frac{1}{2}(\partial \sigma)^{2}-\frac{1}{2} m_{s}^{2} \sigma^{2}-2 \dot{\phi} \eta_{\perp} \sigma \dot{\zeta}^{2},
$$

where $\zeta$ is the adiabatic curvature perturbation, $\dot{\phi}=\left(G_{a b} \dot{\phi}^{a} \dot{\phi}^{b}\right)^{1 / 2}$ is the background velocity in the adiabatic direction and $\sigma$ the entropic field, i.e. the perturbation orthogonal to the background trajectory. The Lagrangian in eq. (2.2) is characterized by 3 parameters: the slow-roll parameter $\epsilon=-\dot{H}^{2} / H^{2}$, the effective entropic mass $m_{s}$ defined in eq. (1.1) and $\eta_{\perp}$. As usual, when $\left|m_{s}^{2}\right| \gg H^{2}$ one can integrate out $\sigma$ and define an EFT for modes below the cutoff $k / a \lesssim\left|m_{s}\right|$. Namely, for those modes, the equation of motion for $\sigma$ reads

$$
\sigma \simeq-2 \frac{\dot{\phi} \eta_{\perp}}{m_{s}^{2}} \dot{\zeta}
$$

Substituting this equality in eq. (2.2) then yields the single-field EFT [13, 30]

$$
\mathcal{L}=\epsilon M_{p}^{2}\left[\frac{\dot{\zeta}^{2}}{c_{s}^{2}}+k^{2} \zeta^{2}\right],
$$

where $c_{s}^{-2}=1+4 \eta_{\perp}^{2} H^{2} / m_{s}^{2}$ is the effective speed of sound. If $m_{s}^{2}+4 \eta_{\perp}^{2} H^{2}>0$ the entropic direction decays on superhorizon scales thus ensuring the stability of the system. However, the effective speed of sound becomes imaginary, $c_{s}^{2}<0$, leading to an exponential growth in the time interval $H \lesssim\left|c_{s}\right| k / a \lesssim\left|m_{s}\right|$. This can be seen more explicitly by solving the equation of motion for $\zeta$ in the constant $c_{s}^{2}$ approximation which yields ${ }^{2}$ [30]

$$
\zeta_{k}(\tau)=\frac{\beta e^{2 x}}{k^{3 / 2}}\left[-f(k, \tau)+\rho e^{i \psi-2 x} g(k, \tau)\right]
$$

\footnotetext{
${ }^{1}$ These statements can be generalized to a system with several entropic directions as long as they are all large and negative [18].

${ }^{2}$ Note that I added an extra (-) sign compared to the solution in [30] in order to make the real and imaginary parts of $\zeta$ positive on superhorizon scales.
} 
where $f$ and $g$ are real functions given by

$$
f(k, \tau)=e^{k\left|c_{s}\right| \tau}\left(k\left|c_{s}\right| \tau-1\right), \quad g(k, \tau)=-f(k,-\tau),
$$

and $x, \beta, \rho, \psi$ are real parameters that need to be computed in the full theory which I assume to be approximately scale-invariant. On super horizon scales, for $\rho e^{-2 x} \ll 1$ the power spectrum is basically determined by the first term in eq. (2.5)

$$
P_{\zeta} \simeq \frac{\beta^{2} e^{2 x}}{2 \pi^{2}}
$$

By imposing the Wronskian condition on the solution in eq. (2.5) one further finds the relation [30]

$$
\beta^{2} \rho \sin (\theta)=\pi^{2} P_{\zeta, 0}
$$

where $P_{\zeta, 0}=H^{2} /\left(8 \pi^{2} \epsilon\left|c_{s}\right| M_{p}^{2}\right)$ is the power spectrum in standard single-field inflation. Fortunately, the leading contributions to non-Gaussianities in the large $x$ limit are proportional to powers of $\beta^{2} \rho \sin (\theta)$. Therefore, the results will only depend only on $\alpha$ and $\left|c_{s}\right|$. In light of that, and without loss of generality, I fix $\rho=1, \psi=\pi / 2$ and use instead

$$
\zeta_{k}(\tau)=\sqrt{\frac{2 \pi^{2} P_{\zeta}}{k^{3}}}\left[-f(k, \tau)+i e^{-2 x} g(k, \tau)\right]
$$

with $P_{\zeta}=P_{\zeta, 0} e^{2 x} / 2$. The parameter $x$ cannot be computed within the EFT. However, if all fields become approximately massless and weakly coupled on scales smaller than the cutoff $\left|c_{s}\right| k / a \lesssim\left|m_{s}\right|$, one expects to recover plane wave solutions at those scales and so to have $x \sim \alpha$. Nevertheless, the precise relation between $x, \alpha$ and $c_{s}$ will depend on the model. For example, in the case of rapid-turn attractors (see app. A), where the turning rate in field-space is $\omega$ and the entropic mass is $m_{s}^{2} \simeq(\xi-1) \omega^{2} H^{2}$, numerical and analytical studies have shown that $x \simeq(2-\sqrt{3+\xi}) \pi \omega / 2$ and $\left|c_{s}\right|=\sqrt{(1-\xi) /(3+\xi)}$ for $\xi<1$ [12, 31]. In particular, for hyperinflation $c_{s}^{2}=\xi=-1$ [11].

Before proceeding to the computation of non-Gaussianities it is useful to introduce the variable $\mathcal{R}$ defined as

$$
\mathcal{R} \equiv \frac{\zeta}{\sqrt{\pi^{2} P_{\zeta, 0}}}, \quad\left\langle\mathcal{R}_{k} \mathcal{R}_{q}\right\rangle=\frac{2 P_{\zeta}}{P_{\zeta, 0} k^{3}} \delta^{(3)}(k+q),
$$

which allows to directly extract the relevant quantities from the mode functions and from the interaction Hamiltonian. In particular note that on superhorizon scales

$$
\operatorname{Re}\left[\mathcal{R}_{k}(0)\right]=\sqrt{\frac{2 P_{\zeta}}{P_{\zeta, 0} k^{3}}}, \quad \operatorname{Im}\left[\mathcal{R}_{k}(0)\right]=\sqrt{\frac{P_{\zeta, 0}}{2 P_{\zeta} k^{3}}} .
$$

\section{Non-Gaussianities}

The shape and size of non-Gaussianities are intrinsic characteristics of a given inflationary model. Interestingly, in the context of the EFT described in the previous section it has been shown that the non-Gaussian parameters have a polynomial, rather than exponential, 
Figure 1: Flattened configurations

dependence on $m_{s}$ in the $\left|m_{s}\right| \gg H$ limit, or equivalently, when $x, \alpha \gg 1^{3}$ [30-32]. Moreover, they peak in shapes where all the momenta collapse to a line (see fig. 1). This characteristic, typical of theories with excited initial states [33, 34], is easy to understand by looking at the time integrals in the in-in formalism. More concretely, the n-point function is given by $[35,36]$

$$
\left\langle\zeta^{n}(\tau)\right\rangle=\sum_{k=0}^{\infty} i^{k} \int_{-\infty}^{\tau} d \tau_{1} \ldots \int_{-\infty}^{\tau_{k-1}} d \tau_{k}\left\langle\left[H_{I}\left(\tau_{k}\right), \ldots\left[H_{I}\left(\tau_{1}\right), \zeta^{n}(\tau)\right] \ldots\right]\right\rangle,
$$

where $H_{I}$ is the interaction Hamiltonian. As the dynamic is effectively described by a singlefield model of inflation, the interactions will be the same as those derived in the context of the EFT of single-field inflation [23].

After inserting the UV cutoff of the EFT, the time integrals become of the form

$$
\mathcal{F}[n, p] \equiv \int_{\alpha}^{0} d y y^{n} \exp [-p y]=-\left.p^{-1-n} \Gamma[n+1, p y]\right|_{y=\alpha} ^{y=0}
$$

where $\Gamma[n, x]$ is the incomplete Gamma function, $n>-1$ and $p>0$ is a function of the different momenta. The integrand peaks at $y_{\max }=n / p$. Therefore, there are basically two distinct classes of shapes:

- (near)-collapsed shapes where $p \ll n / \alpha$ : UV sensitive;

- other shapes: $U V$ and IR finite.

The UV sensitivity is not necessarily dramatic. If the theory behaves approximately as a weakly coupled system of massless fields for scales above the cutoff, $k / a>\left|m_{s}\right|$, as it is likely the case (see eq. (2.2)), the mode functions will quickly be well described by rapidly oscillating plane waves. The oscillatory behavior will then strongly suppress the contribution of the UV to the integrals. Therefore, the results here presented for those shapes can still be trusted at the order of magnitude level. The fact that the non-Gaussianity peaks at that collapsed shapes is insensitive to this discussion.

In the next sections, I will compute the non-Gaussianities for a generic shape and then particularize the results for the equilateral $\left(k_{i}=k\right)$ and flattened $\left(k_{1}=k_{2}+k_{3}\right.$ for the bispectrum and $k_{1}=k_{2}+k_{3}+k_{4}$ for the trispectrum) configurations as representative shapes of those two classes.

\subsection{Bispectrum}

I start by reviewing the bispectrum computation [30, 32]. In Fourier space the 3-point function can generically be written as

$$
\begin{aligned}
\left\langle\zeta_{\mathbf{k}_{1}} \zeta_{\mathbf{k}_{2}} \zeta_{\mathbf{k}_{3}}\right\rangle & =(2 \pi)^{-3 / 2} \delta^{(3)}\left(\mathbf{k}_{1}+\mathbf{k}_{2}+\mathbf{k}_{3}\right) B\left(\mathbf{k}_{1}, \mathbf{k}_{2}, \mathbf{k}_{3}\right) \\
B\left(\mathbf{k}_{1}, \mathbf{k}_{2}, \mathbf{k}_{3}\right) & \equiv(2 \pi)^{4} \frac{S\left(\mathbf{k}_{1}, \mathbf{k}_{2}, \mathbf{k}_{3}\right)}{\left(k_{1} k_{2} k_{3}\right)^{2}} P_{\zeta}^{2}
\end{aligned}
$$

\footnotetext{
${ }^{3}$ I will always work in this limit throughout this section. The subleading corrections in $x$ are suppressed by $e^{-2 x}$ while the subleading terms in $\alpha$ are suppressed by $\alpha^{-1}$.
} 
where $B\left(\mathbf{k}_{1}, \mathbf{k}_{2}, \mathbf{k}_{3}\right)$ is the bispectrum and I have defined the shape function $S\left(\mathbf{k}_{1}, \mathbf{k}_{2}, \mathbf{k}_{3}\right)$ for later convenience. For $n=3$, the first term in eq. (3.1) is

$$
\left\langle\zeta^{3}(\tau)\right\rangle=i \int_{-\infty}^{\tau} d \tau_{1}\left\langle\left[H_{I, 3}\left(\tau_{1}\right), \zeta^{3}(\tau)\right]\right\rangle=-2 \int_{-\infty}^{\tau} d \tau_{1} \operatorname{Im}\left[\left\langle H_{I, 3}\left(\tau_{1}\right) \zeta^{3}(\tau)\right\rangle\right]
$$

where $H_{I, 3}$ is the interaction Hamiltonian of cubic order given in eq. (B.1). The fact that only the imaginary part contributes is crucial to tame the exponential growth of non-Gaussianities $[31,37]$. A similar thing happens in the 4-point function.

I am interested in the late time limit, $\tau \rightarrow 0$. Then, after Fourier transforming the fields using eq. (B.3), performing the Wick contractions, changing variable to $\mathcal{R}$ using eq. (2.10) and using eqs. (B.4) and (2.11) the 3-point function becomes

$$
\begin{aligned}
& \left\langle\zeta_{k_{1}} \zeta_{k_{2}} \zeta_{k_{3}}\right\rangle^{\prime} \simeq \\
& \quad-2 h\left(\frac{\pi^{2} P_{\zeta, 0}}{k_{1} k_{2} k_{3}}\right)^{3 / 2} \int_{-\infty}^{\tau} \frac{d \tau_{1}}{\tau_{1}}\left[\left(\frac{2 P_{\zeta}}{P_{\zeta, 0}}\right)^{3 / 2} \operatorname{Im}\left[\frac{A}{\left|c_{s}^{2}\right|} \mathcal{R}_{k_{1}}^{\prime} \mathcal{R}_{k_{2}}^{\prime} \mathcal{R}_{k_{3}}^{\prime}+\left(\mathbf{k}_{1} \cdot \mathbf{k}_{2}\right) \mathcal{R}_{k_{1}} \mathcal{R}_{k_{2}} \mathcal{R}_{k_{3}}^{\prime}\right]\right. \\
& \left.\quad-3\left(\frac{2 P_{\zeta}}{P_{\zeta, 0}}\right)^{1 / 2} \operatorname{Re}\left[\frac{A}{\left|c_{s}\right|^{2}} \mathcal{R}_{k_{1}}^{\prime} \mathcal{R}_{k_{2}}^{\prime} \mathcal{R}_{k_{3}}^{\prime}+\left(\mathbf{k}_{1} \cdot \mathbf{k}_{2}\right) \mathcal{R}_{k_{1}} \mathcal{R}_{k_{2}} \mathcal{R}_{k_{3}}^{\prime}\right]\right]+5 \text { perm. }
\end{aligned}
$$

The factor of 3 in the last term comes from the 3 different options to chose the imaginary part of the external legs and the minus sign from the complex conjugation. The prime in the correlator denotes that the momentum conserving delta function was suppressed.

Now I can evaluate the integrals using the mode functions in eq. (2.5). The EFT is only valid for modes below the cutoff. Therefore, the time integrals are cutted off at $-k_{\max } \tau_{\mathrm{UV}}\left|c_{s}\right|=\alpha$ where $k_{\max }$ is the largest momentum in the correlator ${ }^{4}$. The results below agree with those found in [30] and show that bispectrum peaks in flattened shapes and is proportional to $\alpha^{3}$.

\subsubsection{Terms in $\zeta^{\prime 3}$}

As mentioned before, the imaginary part ensures that the result is proportional to $e^{4 x}$ thus making the 3-point function proportional to $P_{\zeta}^{2}$ in the $x \gg 1$ limit. In terms of $y=-k_{1}\left|c_{s}\right| \tau$, the terms in $\zeta^{\prime 3}$ in eq. (3.6) simplify to

$$
\begin{aligned}
& \left\langle\zeta_{k_{1}} \zeta_{k_{2}} \zeta_{k_{3}}\right\rangle_{\zeta^{\prime 3}}^{\prime} \simeq \\
& \quad-(2 \pi)^{5 / 2} \frac{P_{\zeta}^{2}}{\left(k_{1} k_{2} k_{3}\right)^{3}}\left(\frac{1}{\left|c_{s}^{2}\right|}+1\right) \frac{3}{8\left|c_{s}\right|} \frac{A}{\left|c_{s}^{2}\right|} \int_{\alpha}^{0} \frac{d y}{y}\left[\left(g_{k_{1}}^{\prime} f_{k_{2}}^{\prime} f_{k_{3}}^{\prime}+2 \text { perm. }\right)+3 f_{k_{1}}^{\prime} f_{k_{2}}^{\prime} f_{k_{3}}^{\prime}\right],
\end{aligned}
$$

where I have used eqs. (B.5) and (2.5). After evaluating the integrals and using eq. (3.2) the shape function in eq. (3.4) becomes

$$
S\left(k_{1}, k_{2}, k_{3}\right)_{\zeta^{\prime 3}}=-\frac{3 A}{8\left|c_{s}\right|^{3}}\left(\frac{1}{\left|c_{s}^{2}\right|}+1\right) \frac{1}{k_{1} k_{2} k_{3}}\left[\left[\frac{k_{2}^{2} k_{3}^{2}\left|c_{s}\right|^{3}}{k_{1}} \mathcal{F}[3, p]+2 \text { perm. }\right]-3 \mathcal{F}\left[3, p_{t}\right]\right],(3.8
$$

where $p=\left(-k_{1}+k_{2}+k_{3}\right) / k_{1}$ and $p_{t}=\left(k_{1}+k_{2}+k_{3}\right) / k_{1}$. As anticipated in the beginning of the section, there are essentially two distinct classes of shapes. For the dominant shapes,

\footnotetext{
${ }^{4} \mathrm{I}$ will assume $k_{1}$ to be the largest momenta throughout the paper apart from sec. 3.2 .2 where I choose $k_{4}$ instead.
} 
near the flattened limit $\alpha p \ll 1$ (c.f. fig. 1), it gives

$$
S\left(k_{1}, k_{2}, k_{3}\right)_{\zeta^{\prime 3}}^{\text {near-flat }}=\frac{A}{8}\left(\frac{1}{\left|c_{s}^{2}\right|}+1\right) \frac{k_{2} k_{3}}{k_{1}^{2}} \alpha^{3}\left[1-\frac{3}{4} p \alpha+\mathcal{O}(p \alpha)^{2}\right] .
$$

Note that the 2 permutations did not contribute to leading order in $\alpha$. For example, for $k_{1} / 2=k_{2}=k_{3}=k$ it yields

$$
S(2 k, k, k)_{\zeta^{\prime 3}} \simeq \frac{A}{32}\left(\frac{1}{\left|c_{s}^{2}\right|}+1\right) \alpha^{3} .
$$

For the remaining shapes the result converges in the $\alpha \gg 1$ limit yielding

$$
S\left(k_{1}, k_{2}, k_{3}\right)_{\zeta^{\prime 3}}^{\text {non-flat }} \simeq-\frac{3}{4} A\left(\frac{1}{\left|c_{s}^{2}\right|}+1\right) \frac{1}{k_{1} k_{2} k_{3}}\left[-\left(\frac{k_{2}^{2} k_{3}^{2}}{k_{1}} \frac{1}{p^{3}}+2 \text { perm. }\right)+\frac{3}{p_{t}^{3}}\right] .
$$

In particular, in the equilateral limit $k_{1}=k_{2}=k_{3}$ it gives

$$
S(k, k, k)_{\zeta^{\prime 3}} \simeq \frac{13}{6} A\left(\frac{1}{\left|c_{s}^{2}\right|}+1\right) .
$$

\subsubsection{Terms in $\zeta^{\prime}(\partial \zeta)^{2}$}

Regarding the terms in $\zeta^{\prime}(\partial \zeta)^{2}$ in eq. (3.6), after using eq. (2.5), I get that

$$
\begin{aligned}
\left\langle\zeta_{k_{1}} \zeta_{k_{2}} \zeta_{k_{3}}\right\rangle_{\zeta^{\prime}(\partial \zeta)^{2}}^{\prime} \simeq & -(2 \pi)^{5 / 2} \frac{P_{\zeta}^{2}}{\left(k_{1} k_{2} k_{3}\right)^{3}}\left(\frac{1}{\left|c_{s}^{2}\right|}+1\right) \frac{\left(\mathbf{k}_{1} \cdot \mathbf{k}_{2}\right)}{16\left|c_{s}\right|}\left[\int_{\alpha}^{0} \frac{d y}{y}\left[g_{k_{1}} f_{k_{2}} f_{k_{3}}^{\prime}+2 \text { perm. }\right]+\right. \\
& \left.+3 f_{k_{1}} f_{k_{2}} f_{k_{3}}^{\prime}\right]+5 \text { perm. }
\end{aligned}
$$

The shape function is in this case given by

$$
\begin{aligned}
& S\left(k_{1}, k_{2}, k_{3}\right)_{\zeta^{\prime}(\partial \zeta)^{2}}= \\
& \quad-\frac{\left(\mathbf{k}_{1} \cdot \mathbf{k}_{2}\right)}{16}\left(\frac{1}{\left|c_{s}^{2}\right|}+1\right) \frac{1}{k_{1} k_{2} k_{3}}\left[\left(\frac{k_{3}^{2}}{k_{1}^{2}}\left(k_{1} \mathcal{F}[1, p]+\left(k_{2}-k_{1}\right) \mathcal{F}[2, p]-k_{2} \mathcal{F}[3, p]\right)\right.\right. \\
& \left.\quad+2 \text { perm. })-3 \frac{k_{3}^{2}}{k_{1}^{2}}\left(k_{2} \mathcal{F}\left[3, p_{t}\right]+\left(k_{2}+k_{1}\right) \mathcal{F}\left[2, p_{t}\right]+k_{1} \mathcal{F}[1, p]\right)\right]+5 \text { perm. }
\end{aligned}
$$

For near-flattened shapes, $p \alpha \ll 1$, in the large- $\alpha$ limit it simplifies to

$$
S\left(k_{1}, k_{2}, k_{3}\right)_{\zeta^{\prime}(\partial \zeta)^{2}}^{\text {near-flat }} \simeq-\frac{\left(\mathbf{k}_{1} \cdot \mathbf{k}_{2}\right)}{48}\left(\frac{1}{\left|c_{s}^{2}\right|}+1\right) \alpha^{3} \frac{k_{3}}{k_{1}^{3}}\left[1-\frac{3}{4} p \alpha+\mathcal{O}(p \alpha)^{2}\right]+5 \text { perm. }(3
$$

In such configuration $\mathbf{k}_{1} \cdot \mathbf{k}_{2}=-k_{1} k_{2}$ and $\mathbf{k}_{2} \cdot \mathbf{k}_{3}=k_{2} k_{3}$ which implies that

$$
S(2 k, k, k)_{\zeta^{\prime}(\partial \zeta)^{2}} \simeq\left(\frac{1}{\left|c_{s}^{2}\right|}+1\right) \frac{\alpha^{3}}{32} .
$$

For other shapes, it gives instead

$$
\begin{aligned}
S\left(k_{1}, k_{2}, k_{3}\right)_{\zeta^{\prime}(\partial \zeta)^{2}}^{\text {non-flat }}= & \frac{\left(\mathbf{k}_{1} \cdot \mathbf{k}_{2}\right)}{16}\left(\frac{1}{\left|c_{s}^{2}\right|}+1\right) \frac{1}{k_{1} k_{2} k_{3}}\left[\left(\frac{k_{3}^{2}}{k_{1}^{2} p^{3}}\left(k_{1}(p-1) p+k_{2}(p-2)\right)\right.\right. \\
& \left.+2 \text { perm. })-3 \frac{k_{3}^{2}}{k_{1}^{2} p_{t}^{3}}\left(k_{1} p_{t}\left(p_{t}+1\right)+k_{2}\left(p_{t}+2\right)\right)\right]+5 \text { perm. }
\end{aligned}
$$

which, in the equilateral limit, reduces to

$$
S(k, k, k)=-\frac{5}{24}\left(\frac{1}{\left|c_{s}^{2}\right|}+1\right) .
$$



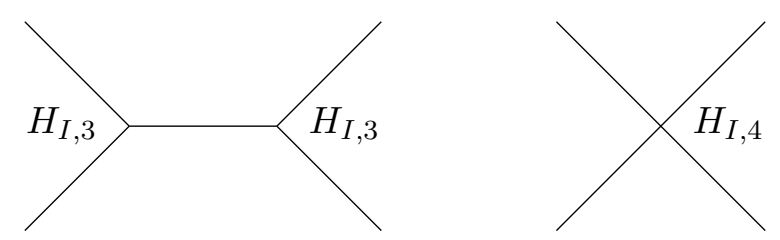

Figure 2: Contributions to the 4-point function: exchange diagram and contact interaction.

\subsection{Trispectrum}

The next step is to compute the contribution to the trispectrum from the two diagrams in fig. 2. The results will share many similarities with the bispectrum calculation: they peak in shapes where all the momenta are (anti-)collinear, which in the case of the exchange diagram does not correspond solely to the $k_{1} \rightarrow k_{2}+k_{3}+k_{4}$ limit, and the remaining shapes are UV and IR convergent in the $\alpha \gg 1$ limit. Moreover, I find that the contribution from the leading flattened shapes scale as $\alpha^{6}$ for the exchange diagram, in agreement with [32], and so dominates over the contact interaction where they scale as $\alpha^{5}$. This can be easily understood from the time integrals in the in-in formalism which in the flattened limit read $\left(\int_{0}^{\alpha} y^{2} d y\right)^{2}$ for the exchange diagram and $\int_{0}^{\alpha} y^{4} d y$ for the contact term .

The two diagrams in fig. 2 correspond to the first two corrections in the in-in expansion and are given by

$$
\begin{aligned}
\left\langle\zeta^{4}(\tau)\right\rangle_{\mathrm{conn} .} \simeq & i \int_{-\infty}^{\tau} d \tau_{1}\left\langle\left[H_{I, 4}\left(\tau_{1}\right), \zeta^{4}(\tau)\right]\right\rangle- \\
& -\int_{-\infty}^{\tau} d \tau_{1} \int_{-\infty}^{\tau_{1}} d \tau_{2}\left\langle\left[H_{I, 3}\left(\tau_{2}\right),\left[H_{I, 3}\left(\tau_{1}\right), \zeta^{4}(\tau)\right]\right]\right\rangle \equiv\left\langle\zeta^{4}(\tau)\right\rangle_{c, 1}+\left\langle\zeta^{4}(\tau)\right\rangle_{c, 2}
\end{aligned}
$$

where $H_{I, 4}$ is the interaction Hamiltonian of quartic order given in eq. (B.8). In Fourier space the 4-point function is generically given by

$$
\left\langle\zeta_{k_{1}} \zeta_{k_{2}} \zeta_{k_{3}} \zeta_{k_{4}}\right\rangle_{\text {conn. }}=\frac{(2 \pi)^{3} P_{\zeta}^{3}}{\left(k_{1} k_{2} k_{3} k_{4}\right)^{9 / 4}} \mathcal{T}\left(k_{1}, k_{2}, k_{3}, k_{4}\right) \delta^{(3)}\left(\sum_{\mathbf{i}} \mathbf{k}_{\mathbf{i}}\right) .
$$

The goal now is to calculate $\mathcal{T}$ from the different contributions.

\subsubsection{Quartic interaction}

We start by computing $\left\langle\zeta^{4}(\tau)\right\rangle_{c, 1}$, the contact interaction. Using eqs. (B.3) and (2.10) the correlator becomes

$$
\left\langle\zeta_{k_{1}} \zeta_{k_{2}} \zeta_{k_{3}} \zeta_{k_{4}}\right\rangle_{c, 1}=-2\left(\pi^{2} P_{\zeta, 0}\right)^{2} \int_{-\infty}^{\tau} d \tau_{1} \operatorname{Im}\left[\left\langle H_{I, 4}\left(\tau_{1}\right),\left(\mathcal{R}_{k_{1}} \mathcal{R}_{k_{2}} \mathcal{R}_{k_{3}} \mathcal{R}_{k_{4}}\right)_{\tau}\right\rangle\right]
$$

Just like in the bispectrum, the imaginary part is crucial to tame exponential growth of nonGaussianities. Now I perform the Wick contractions and separate the integrand in two terms depending on whether I take the real or imaginary part of the external legs. After using eqs. (2.11), (3.20) and inserting the interaction Hamiltonian in eq. (B.9) the trispectrum becomes

$$
\mathcal{T}\left(k_{1}, k_{2}, k_{3}, k_{4}\right)=\frac{1}{32\left|c_{s}\right|}\left(\frac{1}{\left|c_{s}^{2}\right|}+1\right) \frac{1}{\left(k_{1} k_{2} k_{3} k_{4}\right)^{3 / 4}} \sum_{i=1}^{3} \mathcal{I}_{i}\left(k_{1}, k_{2}, k_{3}, k_{4}\right),
$$


where

$$
\begin{aligned}
\mathcal{I}_{1}\left(k_{1}, k_{2}, k_{3}, k_{4}\right) \simeq & 24 B \int_{\alpha}^{0} \frac{d y}{-k_{1}\left|c_{s}\right|}\left[-\left[g_{k_{1}}^{\prime} f_{k_{2}}^{\prime} f_{k_{3}}^{\prime} f_{k_{4}}^{\prime}+3 \text { perm. }\right]-f_{k_{1}}^{\prime} f_{k_{2}}^{\prime} f_{k_{3}}^{\prime} f_{k_{4}}^{\prime}\right], \\
\mathcal{I}_{2}\left(k_{1}, k_{2}, k_{3}, k_{4}\right) \simeq & -4 C\left(\mathbf{k}_{1} \cdot \mathbf{k}_{2}\right) \int_{\alpha}^{0} \frac{d y}{-k_{1}\left|c_{s}\right|}\left[-\left[g_{k_{1}} f_{k_{2}} f_{k_{3}}^{\prime} f_{k_{4}}^{\prime}+3 \text { perm. }\right]-\right. \\
& \left.-f_{k_{1}} f_{k_{2}} f_{k_{3}}^{\prime} f_{k_{4}}^{\prime}\right]+5 \text { perm. }, \\
\mathcal{I}_{3}\left(k_{1}, k_{2}, k_{3}, k_{4}\right) \simeq & \left|c_{s}^{2}\right|\left(\mathbf{k}_{1} \cdot \mathbf{k}_{2}\right)\left(\mathbf{k}_{3} \cdot \mathbf{k}_{4}\right) \int_{\alpha}^{0} \frac{d y}{-k_{1}\left|c_{s}\right|}\left[-\left[g_{k_{1}} f_{k_{2}} f_{k_{3}} f_{k_{4}}+3 \text { perm. }\right]-\right. \\
& \left.-f_{k_{1}} f_{k_{2}} f_{k_{3}} f_{k_{4}}\right]+5 \text { perm. }
\end{aligned}
$$

In the last step, I inserted the mode functions in eq. (2.5), changed the time variable to $y=-k_{1} \tau\left|c_{s}\right|$ and inserted the EFT cutoff in the time integral assuming $k_{1}$ to be the largest momenta.

Terms in $\zeta^{\prime 4}$ : Using eqs. (3.2) and (2.9) the integral in eq. (3.23) simplifies to

$$
\mathcal{I}_{1}\left(k_{1}, k_{2}, k_{3}, k_{4}\right)=-24 B\left|c_{s}^{3}\right| \frac{\left(k_{2} k_{3} k_{4}\right)^{2}}{k_{1}^{3}}\left[\mathcal{F}[4, q y]+3 \text { perm. }-\mathcal{F}\left[4, q_{t} y\right]\right]
$$

where $q \equiv\left(-k_{1}+k_{2}+k_{3}+k_{4}\right) / k_{1}$ and $q_{t}=\left(k_{1}+k_{2}+k_{3}+k_{4}\right) / k_{1}$. The 3 permutations are similar, up to the appropriate redefinition of $q$. However, they do not contribute to the flattened limit, $q \ll 1 / \alpha$, to leading order in $\alpha$ which reads

$$
\mathcal{I}_{1}^{\text {near-flat }}\left(k_{1}, k_{2}, k_{3}, k_{4}\right)=24 B \alpha^{5}\left|c_{s}\right|^{3} \frac{\left(k_{2} k_{3} k_{4}\right)^{2}}{k_{1}^{3}}\left(\frac{1}{5}-\frac{1}{6} q \alpha+\mathcal{O}(q \alpha)^{2}\right)
$$

and for $k_{1} / 3=k_{2}=k_{3}=k_{4}=k$ yields the trispectrum

$$
\mathcal{T}_{1}(3 k, k, k, k)=\frac{B\left|c_{s}\right|^{2}}{180 \cdot 3^{3 / 4}}\left(\frac{1}{\left|c_{s}^{2}\right|}+1\right) \alpha^{5} .
$$

The remaining shapes are UV and IR convergent in the $\alpha \gg 1$ limit and given by

$$
\mathcal{I}_{1}^{\text {non-flat }}\left(k_{1}, k_{2}, k_{3}, k_{4}\right)=24^{2} B\left|c_{s}\right|^{3}\left[\frac{\left(k_{2} k_{3} k_{4}\right)^{2}}{k_{1}^{3} q^{5}}+3 \text { perm. }-\frac{\left(k_{2} k_{3} k_{4}\right)^{2}}{k_{1}^{3} q_{t}^{5}}\right] .
$$

In particular, for equilateral shapes

$$
\mathcal{T}_{1}(k, k, k, k)=\frac{855}{512} B\left|c_{s}\right|^{2} .
$$

Terms in $\zeta^{\prime 2}(\partial \zeta)^{2}$ : Following steps similar to those of above, $\mathcal{I}_{2}$ in eq. (3.24) evaluates to

$$
\begin{aligned}
\mathcal{I}_{2}\left(k_{1}, k_{2}, k_{3}, k_{4}\right)= & -4 C\left|c_{s}\right|\left(\mathbf{k}_{1} \cdot \mathbf{k}_{2}\right) \frac{\left(k_{3} k_{4}\right)^{2}}{k_{1}^{4}} \times \\
& {\left[\left[k_{2} \mathcal{F}[4, q y]+\left(k_{1}-k_{2}\right) \mathcal{F}[3, q y]-k_{1} \mathcal{F}[2, q y]+3 \text { perm. }\right]\right.} \\
& \left.+\left[k_{2} \mathcal{F}\left[4, q_{t} y\right]+\left(k_{1}+k_{2}\right) \mathcal{F}\left[3, q_{t} y\right]+k_{1} \mathcal{F}\left[2, q_{t} y\right]\right]\right]+5 \text { perm. }
\end{aligned}
$$


In the flattened limit, $q \ll 1 / \alpha$, the leading terms are

$$
\begin{aligned}
& \mathcal{I}_{2}^{\text {near-flat }}\left(k_{1}, k_{2}, k_{3}, k_{4}\right)=4 C\left|c_{s}\right| \times \\
& \quad\left[\left(\mathbf{k}_{1} \cdot \mathbf{k}_{2}\right) \frac{k_{3}^{2} k_{4}^{2}}{60 k_{1}^{4}} \alpha^{3}\left(5\left(k_{3}+k_{4}\right)(3 \alpha-4)+4 k_{2}\left(3 \alpha^{2}-5\right)\right)+5 \text { perm. }\right]+\mathcal{O}(q \alpha) .
\end{aligned}
$$

When $q \rightarrow 0$ all momenta are collinear apart from $k_{1}$ which is anti-collinear. Therefore, for $\alpha \gg 1$ the trispectrum is given by

$$
\mathcal{T}_{2}(3 k, k, k, k)=-\frac{C}{180 \cdot 3^{3 / 4}}\left(\frac{1}{\left|c_{s}^{2}\right|}+1\right) \alpha^{5} .
$$

For other shapes, in the $\alpha \gg 1$ limit, I get instead

$$
\begin{aligned}
\mathcal{I}_{2}^{\text {non-flat }}\left(k_{1}, k_{2}, k_{3}, k_{4}\right)= & -8\left(\mathbf{k}_{1} \cdot \mathbf{k}_{2}\right) C\left|c_{s}\right| \frac{k_{3}^{2} k_{4}^{2}}{k_{1}^{4}}\left[\frac{1}{q^{5}}\left(\left(k_{1}(q-3) q+3 k_{2}(q-4)\right)+3 \text { perm. }\right)-\right. \\
& \left.\frac{1}{q_{t}^{5}}\left(k_{1} q_{t}\left(q_{t}+3\right)+3 k_{2}\left(q_{t}+4\right)\right)\right]+5 \text { perm. }
\end{aligned}
$$

Terms in $(\partial \zeta)^{4}$ : Finally I look at $\mathcal{I}_{3}$ in eq. (3.25). After inserting the mode functions in eq. (2.5) it reads

$$
\begin{array}{r}
\mathcal{I}_{3}\left(k_{1}, k_{2}, k_{3}, k_{4}\right)=\left|c_{s}\right| \frac{\left(\mathbf{k}_{1} \cdot \mathbf{k}_{2}\right)\left(\mathbf{k}_{3} \cdot \mathbf{k}_{4}\right)}{k_{1}^{4}} \int_{\alpha}^{0} d y\left[(y-1)\left(k_{2} y+k_{1}\right)\left(k_{3} y+k_{1}\right)\left(k_{4} y+k_{1}\right) e^{-q y}\right. \\
\left.+3 \text { perm. }+\frac{1}{k_{1}^{4}\left|c_{s}\right|}(y+1)\left(k_{2} y+k_{1}\right)\left(k_{3} y+k_{1}\right)\left(k_{4} y+k_{1}\right) e^{-y q_{t}}\right]+5 \text { perm. }
\end{array}
$$

The expression in terms of the function $\mathcal{F}$ defined in eq. (3.2) is long and not very informative so I give it in eq. (C.1). In the near-flattened limit, and for $\alpha \gg 1$, it gives

$$
\mathcal{I}_{3}^{\text {near-flat }}\left(k_{1}, k_{2}, k_{3}, k_{4}\right) \simeq-\frac{1}{5}\left|c_{s}\right| \alpha^{5} \frac{k_{2} k_{3} k_{4}}{k_{1}^{4}}\left[\left(\mathbf{k}_{1} \cdot \mathbf{k}_{2}\right)\left(\mathbf{k}_{3} \cdot \mathbf{k}_{4}\right)+5 \text { perm. }\right]+\mathcal{O}(q \alpha),
$$

and yields the trispectrum

$$
\mathcal{T}_{3}(3 k, k, k, k)=\frac{1}{720 \cdot 3^{3 / 4}}\left(\frac{1}{\left|c_{s}^{2}\right|}+1\right) \alpha^{5} .
$$

For other shapes I get instead the result in eq. (C.2).

To sum up, the contributions to the trispectrum from the contact interaction peak in the flattened shape, proportionally to $\alpha^{5}$, while other shapes are $\alpha$-independent in the large- $\alpha$ limit.

\subsubsection{Cubic interaction}

Finally, I pass to the main contribution to the trispectrum, the term $\left\langle\zeta^{4}(\tau)\right\rangle_{c, 2}$ in eq. (3.19) corresponding to the exchange diagram in fig. 2. I start by expanding the integrand into

$$
\left\langle\left[\hat{H}_{I}\left(\tau_{2}\right),\left[\hat{H}_{I}\left(\tau_{1}\right), \hat{\zeta}^{4}(\tau)\right]\right]\right\rangle=2 \operatorname{Re}\left\langle\hat{H}_{I}\left(\tau_{2}\right) \hat{H}_{I}\left(\tau_{1}\right) \hat{\zeta}^{4}(\tau)-\hat{H}_{I}\left(\tau_{2}\right) \hat{\zeta}^{4}(\tau) \hat{H}_{I}\left(\tau_{1}\right)\right\rangle .
$$


Then, after changing from $\zeta$ to $\mathcal{R}$ using eq. (2.10) and inserting the cubic Hamiltonian in eq. (B.4) the 4-point function is given, in Fourier space, by

$$
\begin{aligned}
& \left\langle\zeta_{k_{1}} \zeta_{k_{2}} \zeta_{k_{3}} \zeta_{k_{4}}\right\rangle_{c, 2}=-2 h^{2}\left(\pi^{2} P_{\zeta, 0}\right)^{2} \int_{-\infty}^{\tau} d \tau_{1} \int_{-\infty}^{\tau_{1}} d \tau_{2} \\
& \quad \operatorname{Re}\left\langle\tilde{H}_{I, 3}\left(\tau_{2}\right)\left(\tilde{H}_{I, 3}\left(\tau_{1}\right)\left(\mathcal{R}_{k_{1}} \mathcal{R}_{k_{2}} \mathcal{R}_{k_{3}} \mathcal{R}_{k_{4}}\right)_{\tau}-\left(\mathcal{R}_{k_{1}} \mathcal{R}_{k_{2}} \mathcal{R}_{k_{3}} \mathcal{R}_{k_{4}}\right)_{\tau} \tilde{H}_{I, 3}\left(\tau_{1}\right)\right)\right\rangle .
\end{aligned}
$$

Each $\tilde{H}_{I, 3}$ contains two distinct terms so there are 4 different combinations. I define the functions $\mathcal{K}_{i}$ associated with each of those terms as

$$
\left\langle\zeta_{k_{1}} \zeta_{k_{2}} \zeta_{k_{3}} \zeta_{k_{4}}\right\rangle_{c, 2}=-\frac{\left(\frac{1}{\left|c_{s}^{2}\right|}+1\right)^{2}}{2^{9}\left|c_{s}\right|^{2}} \frac{(2 \pi)^{3} P_{\zeta}^{3}}{\left(k_{1} k_{2} k_{3} k_{4}\right)^{3}} \delta^{(3)}\left(\sum_{i=1}^{4} k_{i}\right) \sum_{i=1}^{4} \mathcal{K}_{i}
$$

where $P_{\zeta}^{3} / 2$ was factored out for convenience. The trispectrum in eq. (3.20) is then related to $\mathcal{K}_{i}$ by

$$
\mathcal{T}\left(k_{1}, k_{2}, k_{3}, k_{4}\right)=-\left(\frac{1}{\left|c_{s}^{2}\right|}+1\right)^{2} \frac{1}{2^{9}\left|c_{s}\right|^{2}} \frac{1}{\left(k_{1} k_{2} k_{3} k_{4}\right)^{3 / 4}} \sum_{i=1}^{4} \mathcal{K}_{i}\left(k_{1}, k_{2}, k_{3}, k_{4}\right) .
$$

The function $\mathcal{K}_{1}$ is associated with two insertions of $\tilde{H}_{3,2}$, defined in eq. (B.4), and it is given by

$$
\begin{gathered}
\mathcal{K}_{1}\left(k_{1}, k_{2}, k_{3}, k_{4}\right)=-2 \times 2 \times(3 \times 3 \times 4)\left(\frac{A}{\left|c_{s}^{2}\right|}\right)^{2}\left(k_{1} k_{2} k_{3} k_{4}\right)^{3} e^{-6 x} \int_{-\infty}^{\tau} \frac{d \tau_{1}}{\tau_{1}} \int_{-\infty}^{\tau_{1}} \frac{d \tau_{2}}{\tau_{2}} \\
\operatorname{Im}\left[\left(\mathcal{R}_{k_{1}}^{\prime} \mathcal{R}_{k_{2}}^{\prime} \mathcal{R}_{k_{12}}^{\prime}\right)_{\tau_{2}}\left(\mathcal{R}_{k_{1}} \mathcal{R}_{k_{2}}\right)_{\tau}^{*} \mathcal{R}_{k_{12}, \tau_{1}}^{\prime *}\right] \operatorname{Im}\left[\left(\mathcal{R}_{k_{4}}^{\prime} \mathcal{R}_{k_{3}}^{\prime}\right)_{\tau 1}\left(\mathcal{R}_{k_{3}} \mathcal{R}_{k_{4}}\right)_{\tau}^{*}\right]+5 \text { perm. }
\end{gathered}
$$

where $\mathbf{k}_{12}=\mathbf{k}_{1}+\mathbf{k}_{2}$. Some of the pre-factors are shared by the several $\mathcal{K}_{i}$ so I explain here their origin. The factor $3 \times 3 \times 4$ comes from permutations within the first vertex times permutations within the second vertex and permutations $1 \leftrightarrow 2,3 \leftrightarrow 4$ in the external legs ${ }^{5}$. The two factors of 2 come from picking one of the imaginary parts and to compensate the factor of $1 / 2$ which I factored out in eq. (3.40). The $\mathcal{K}_{2}$ term is associated with one insertion of each vertex, $\tilde{H}_{3,1}$ and $\tilde{H}_{3,2}$, and is given by

$$
\begin{aligned}
& \mathcal{K}_{2}\left(k_{1}, k_{2}, k_{3}, k_{4}\right)=-4 \times(3 \times 4)\left(\frac{A}{\left|c_{s}^{2}\right|}\right)\left(k_{1} k_{2} k_{3} k_{4}\right)^{3} e^{-6 x} \int_{-\infty}^{\tau} \frac{d \tau_{1}}{\tau_{1}} \int_{-\infty}^{\tau_{1}} \frac{d \tau_{2}}{\tau_{2}} \\
& \quad \operatorname{Im}\left[\left[\left(\mathbf{k}_{1} \cdot \mathbf{k}_{2}\right)\left(\mathcal{R}_{k_{12}}^{\prime} \mathcal{R}_{k_{1}} \mathcal{R}_{k_{2}}\right)_{\tau_{2}} \mathcal{R}_{k_{12}, \tau_{1}}^{\prime *}\left(\mathcal{R}_{k_{1}} \mathcal{R}_{k_{2}}\right)_{\tau}^{*}\right] \operatorname{Im}\left[\left(\mathcal{R}_{k_{4}}^{\prime} \mathcal{R}_{k_{3}}^{\prime}\right)_{\tau_{1}}\left(\mathcal{R}_{k_{3}} \mathcal{R}_{k_{4}}\right)_{\tau}^{*}\right]+\right. \\
& \quad+2 \text { perm. }]+5 \text { perm. }
\end{aligned}
$$

$\mathcal{K}_{3}$ is similar to $\mathcal{K}_{2}$ but with the vertices in the opposite order

$$
\begin{aligned}
& \mathcal{K}_{3}\left(k_{1}, k_{2}, k_{3}, k_{4}\right)=-4 \times(3 \times 4)\left(\frac{A}{\left|c_{s}^{2}\right|}\right)\left(k_{1} k_{2} k_{3} k_{4}\right)^{3} e^{-6 x} \int_{-\infty}^{\tau} \frac{d \tau_{1}}{\tau_{1}} \int_{-\infty}^{\tau_{1}} \frac{d \tau_{2}}{\tau_{2}} \\
& \quad \operatorname{Im}\left[\left(\mathcal{R}_{k_{12}}^{\prime} \mathcal{R}_{k_{3}}^{\prime} \mathcal{R}_{k_{4}}^{\prime}\right)_{\tau_{2}} \mathcal{R}_{k_{12}, \tau_{1}}^{\prime *}\left(\mathcal{R}_{k_{3}} \mathcal{R}_{k_{4}}\right)_{\tau}^{*}\right] \operatorname{Im}\left[\left(\mathbf{k}_{1} \cdot \mathbf{k}_{2}\right)\left(\mathcal{R}_{k_{1}} \mathcal{R}_{k_{2}}\right)_{\tau_{1}}\left(\mathcal{R}_{k_{1}} \mathcal{R}_{k_{2}}\right)_{\tau}^{*}+\right. \\
& \quad+2 \text { perm. }]+5 \text { perm. }
\end{aligned}
$$

\footnotetext{
${ }^{5}$ The previous expression, jointly with eq. (3.41) agrees with eq. (21) of [32].
} 


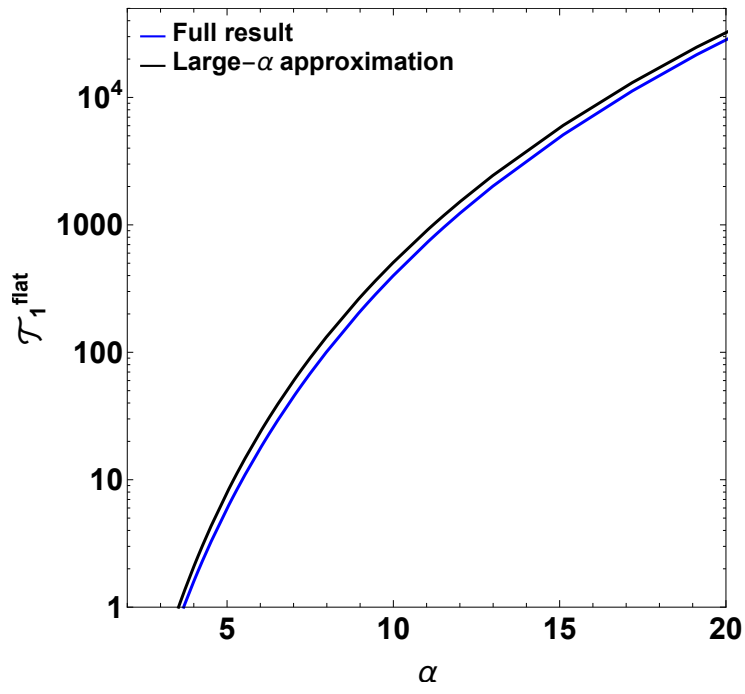

Figure 3: Contribution of $\mathcal{K}_{1}$ to the trispectrum, in the flattened shape and as a function of $\alpha$. I plot the full expression and compare it with the large- $\alpha$ approximation.

Finally, $\mathcal{K}_{4}$ is associated with two insertions of $\tilde{H}_{3,1}$ and given by

$$
\begin{aligned}
& \mathcal{K}_{4}\left(k_{1}, k_{2}, k_{3}, k_{4}\right)=-4 \times 4\left(k_{1} k_{2} k_{3} k_{4}\right)^{3} e^{-6 x} \int_{-\infty}^{\tau} \frac{d \tau_{1}}{\tau_{1}} \int_{-\infty}^{\tau_{1}} \frac{d \tau_{2}}{\tau_{2}} \\
& \quad \operatorname{Im}\left[[ ( \mathbf { k } _ { 1 } \cdot \mathbf { k } _ { 2 } ) ( \mathcal { R } _ { k _ { 1 2 } , \tau _ { 2 } } ^ { \prime } \mathcal { R } _ { k _ { 1 } } \mathcal { R } _ { k _ { 2 } } ) _ { \tau _ { 2 } } \mathcal { R } _ { k _ { 1 2 } , \tau _ { 1 } } ^ { \prime * } ( \mathcal { R } _ { k _ { 1 } } \mathcal { R } _ { k _ { 2 } } ) _ { \tau } ^ { * } ] \operatorname { I m } \left[\left(\mathbf{k}_{3} \cdot \mathbf{k}_{4}\right)\left(\mathcal{R}_{k_{4}} \mathcal{R}_{k_{3}}\right)_{\tau_{1}}\left(\mathcal{R}_{k_{3}} \mathcal{R}_{k_{4}}\right)_{\tau}^{*}\right.\right. \\
& \quad+2 \text { perm. }]+2 \text { perm. }]+5 \text { perm. }
\end{aligned}
$$

Similarly to the previous sections, the time integrals are of the form

$$
\int d \tau_{1} \tau_{1}^{n} e^{\left(k_{1}+k_{2}-k_{12}\right) \tau_{1}} \int d \tau_{2} \tau_{2}^{m} e^{\left(k_{3}+k_{4}-k_{12}\right) \tau_{2}}
$$

and become UV dominated when the exponent becomes smaller than $1 / \alpha$. However, this time such shapes are not only those in the flattened limit, $k_{4} \rightarrow k_{1}+k_{2}+k_{3}$, but also other collapsed shapes where all momenta are still (anti-)collinear. Nevertheless, as all such shapes give similar results I will again choose the flattened limit as representative.

In the previous sections, I have assumed $k_{1}$ to be the largest momenta and introduce the UV cutoff in the variable $y=-k_{1} \tau\left|c_{s}\right|$. For convenience, I assume in this subsection $k_{4}$ to be the largest momenta and define instead $z=-k_{4} \tau\left|c_{s}\right|$.

Terms in $\mathcal{K}_{1}$ : Let me start by the terms in $\mathcal{K}_{1}$ which are the most symmetric. After inserting the mode functions in eqs. (2.5) and (2.9) I get in the large $x$ limit

$$
\begin{gathered}
\mathcal{K}_{1}\left(k_{1}, k_{2}, k_{3}, k_{4}\right)=-144\left(\frac{A}{\left|c_{s}^{2}\right|}\right)^{2} \frac{k_{2}^{2} k_{3}^{2} k_{1}^{2} k_{12}\left|c_{s}\right|^{6}}{k_{4}^{4}} \int_{\alpha}^{0} \frac{d z_{1}}{z_{1}} \int_{\alpha}^{z_{1}} \frac{d z_{2}}{z_{2}} z_{1}^{3} z_{2}^{3} \times \\
\left(e^{\frac{2 k_{3} z_{1}}{k_{4}}}+e^{2 z_{1}}-2\right)\left(e^{\frac{2 k_{1} z_{2}}{k_{4}}}+e^{\frac{2 k_{2} z_{2}}{k_{4}}}+e^{\frac{2 k_{12} z_{2}}{k_{4}}}-e^{\frac{2 k_{12} z_{1}}{k_{4}}}-2\right) e^{-\left(p_{1} z_{2}+p_{2} z_{1}\right)}+5 \text { perm. }
\end{gathered}
$$

where $p_{1} \equiv\left(k_{1}+k_{2}+k_{12}\right) / k_{4}$ and $p_{2} \equiv\left(k_{3}+k_{4}+k_{12}\right) / k_{4}$. The leading shapes are those such that $p_{1}, p_{2} \rightarrow 0$ simultaneously. As mentioned above, such shapes correspond to limits 
where the quadrilateral formed by the 4 momenta collapses. I look at the shape $k_{1}=k_{2}=$ $k_{3}=k_{4} / 3=k$ as representative. In that case, $k_{12}=2 k$ (fig. 1 ) and in large- $\alpha$ limit it gives

$$
\mathcal{K}_{1}^{\text {flat }}(k, k, k, 3 k)=-3 \times \frac{144}{729}\left(\frac{A}{\left|c_{s}^{2}\right|}\right)^{2} \alpha^{6}\left|c_{s}\right|^{6} k^{3},
$$

where the last factor of three comes from the fact that only the permutations of the external legs where $\mathcal{R}_{4}$ is in the innermost commutator contribute to the leading order in $\alpha$. Plugging the factors in eq. (3.41) the trispectrum becomes

$$
\begin{aligned}
\mathcal{T}_{1}^{\text {flat }}(k, k, k, 3 k) & =-\frac{1}{2^{9} 3^{3 / 4}\left|c_{s}\right|^{2} k^{3}}\left(\frac{1}{\left|c_{s}^{2}\right|}+1\right)^{2} \mathcal{K}_{1}^{\text {flat }}(k, k, k, 3 k) \\
& \simeq \frac{A^{2}}{864 \cdot 3^{3 / 4}} \alpha^{6}\left(\frac{1}{\left|c_{s}^{2}\right|}+1\right)^{2} .
\end{aligned}
$$

This result is 3 times larger than the result obtained in [32]. In fig. 3 I show that the large- $\alpha$ expression is indeed a good approximation for the full result.

For the equilateral shape, $k_{1}=k_{2}=k_{3}=k_{4}=k$, the result converges yielding

$$
\begin{aligned}
& \mathcal{K}_{1}^{\text {equi }}(k, k, k, k)=-2 \cdot 144\left(\frac{A}{c_{s}^{2}}\right)^{2} b_{12} k^{3}\left|c_{s}\right|^{6} \times \\
& \quad\left(\frac{4}{b_{12}^{6}}+\frac{4}{\left(b_{12}+2\right)^{6}}-\frac{8}{b_{12}^{3}\left(b_{12}+2\right)^{3}}-b_{12} \frac{93 b_{12}^{4}-1048 b_{12}^{2}+3600}{64\left(4-b_{12}^{2}\right)^{3}}\right)+5 \text { perm. }
\end{aligned}
$$

where $b_{i j}=k_{i j} / k_{4}$ ranges between zero and two. The result diverges precisely in the collinear and anti-collinear limits where the large- $\alpha$ approximation is no longer valid. The remaining 5 permutations correspond to changes in the external legs which amount to swap $b_{12}$ by the remaining 5 possibilities. However, due to momentum conservation there are only 2 independent combinations $\left(b_{12}, b_{14}\right)$ because $b_{12}=b_{34}, b_{13}=b_{24}, b_{14}=b_{23}$ and

$$
k_{13}=\sqrt{k_{1}^{2}+k_{2}^{2}+k_{3}^{2}+k_{4}^{2}-k_{12}^{2}-k_{14}^{2}} .
$$

In terms of $\mathcal{T}$ the equilateral shape yields

$$
\mathcal{T}_{1}^{\text {equi }}(k, k, k, k)=-\left(\frac{1}{\left|c_{s}^{2}\right|}+1\right)^{2} \frac{1}{2^{9} k^{3}\left|c_{s}\right|^{2}} \mathcal{K}_{1}^{\text {equi }}(k, k, k, k)
$$

In fig. $4 \mathrm{I}$ plot the results as a function of $\alpha$ and $b_{12}$. As already mentioned, when $b_{12}$ or $b_{14}$ approach the (anti-) colinear limit the results approach those for the collapsed shapes where the trispectrum grows with $\alpha$. 

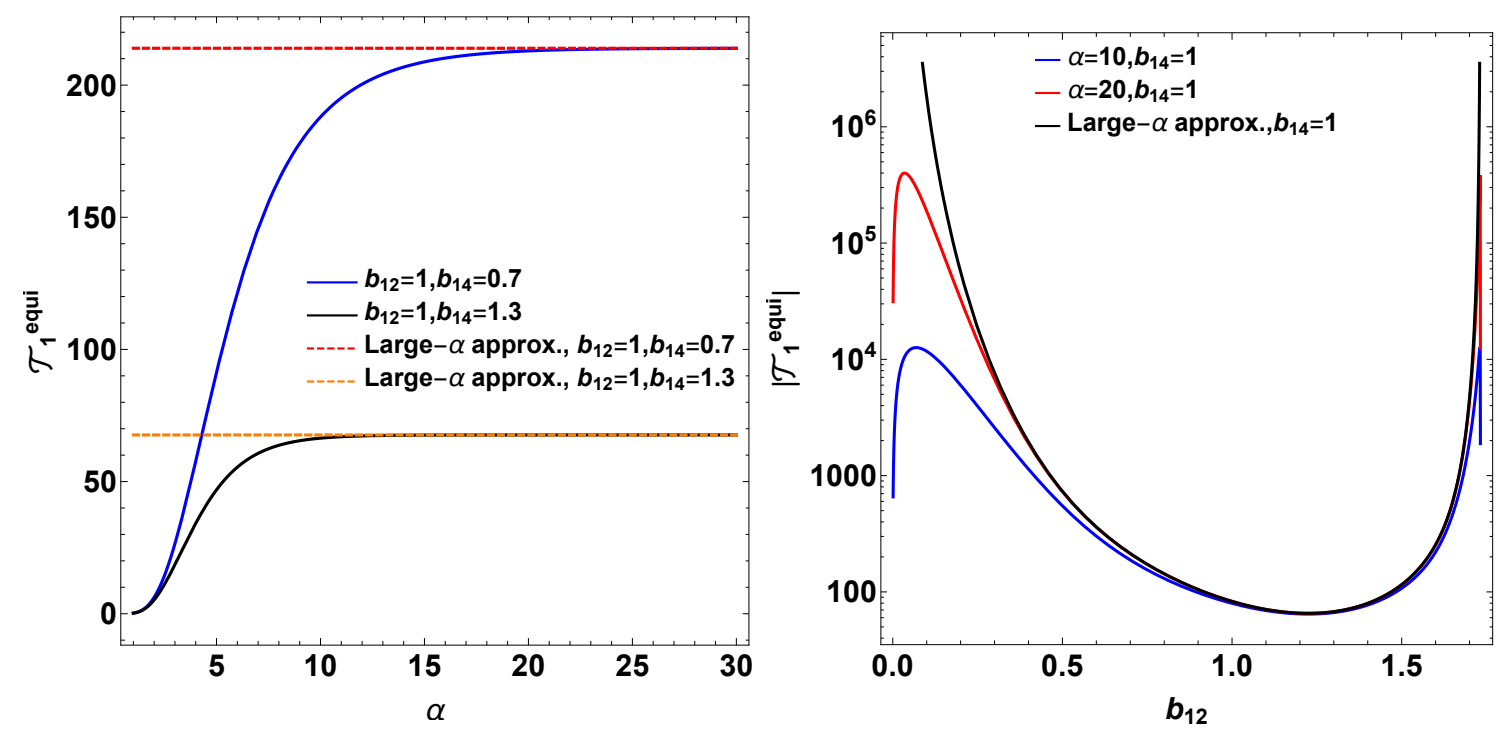

Figure 4: Equilateral trispectrum, $\mathcal{T}_{1}^{\text {equi }}$, as a function of: (left plot) $\alpha$ for 2 different values of $b_{14}$, (right plot) $b_{12}$ for two different values of $\alpha$. I also compare the full expressions with the large- $\alpha$ approximation. I fixed $\left|c_{s}^{2}\right|=1, A=1$ in both plots.

Terms in $\mathcal{K}_{2}$ and $\mathcal{K}_{3}$ : Inserting the mode functions in eqs. (3.43) and (3.44) yields

$$
\begin{aligned}
& \mathcal{K}_{2}\left(k_{1}, k_{2}, k_{3}, k_{4}\right)=48 A\left|c_{s}\right|^{2} \int_{\alpha}^{0} \frac{d z_{1}}{z_{1}} \int_{\alpha}^{z_{1}} \frac{d z_{2}}{z_{2}}\left[\left(\mathbf{k}_{1} \cdot \mathbf{k}_{2}\right) z_{1}^{3} z_{2} \frac{k_{1} k_{2} k_{12} k_{3}^{2}}{k_{4}^{4}} e^{-\left(p_{1} z_{2}+p_{2} z_{1}\right)} \times\right. \\
& \quad\left(e^{\frac{2 k_{3} z_{1}}{k_{4}}}+e^{2 z_{1}}-2\right)\left(\left(k_{2} z_{2}+k_{4}\right)\left(e^{\frac{2 k_{1} z_{2}}{k_{4}}}\left(k_{1} z_{2}-k_{4}\right)+\left(e^{\frac{2 k_{12} z_{1}}{k_{4}}}-e^{\frac{2 k_{12} z_{2}}{k_{4}}}+2\right)\left(k_{1} z_{2}+k_{4}\right)\right)\right. \\
& \left.\left.\quad-e^{\frac{2 k_{2} z_{2}}{k_{4}}}\left(k_{1} z_{2}+k_{4}\right)\left(k_{4}-k_{2} z_{2}\right)\right)+2 \text { perm. }\right]+5 \text { perm. } \\
& \mathcal{K}_{3}\left(k_{1}, k_{2}, k_{3}, k_{4}\right)=48 A\left|c_{s}\right|^{2} \int_{\alpha}^{0} \frac{d z_{1}}{z_{1}} \int_{\alpha}^{z_{1}} \frac{d z_{2}}{z_{2}}\left[\left(\mathbf{k}_{1} \cdot \mathbf{k}_{2}\right) z_{2}^{3} z_{1} \frac{k_{1} k_{2} k_{12} k_{3}^{2}}{k_{4}^{4}} e^{-\left(p_{1} z_{2}+p_{2} z_{2}\right)} \times\right. \\
& \quad \times\left(e^{\frac{2 k_{2} z_{1}}{k_{4}}}\left(k_{1} z_{1}+k_{4}\right)\left(k_{4}-k_{2} z_{1}\right)+\left(k_{2} z_{1}+k_{4}\right)\left(e^{\frac{2 k_{1} z_{1}}{k_{4}}}\left(k_{4}-k_{1} z_{1}\right)-2\left(k_{1} z_{1}+k_{4}\right)\right)\right) \times \\
& \\
& \left.\quad\left(-e^{\frac{2 k_{12} z_{1}}{k_{4}}}+e^{\frac{2 k_{3} z_{2}}{k_{4}}}+e^{\frac{2 k_{12} z_{2}}{k_{4}}}+e^{2 z_{2}}-2\right)+2 \text { perm. }\right]+5 \text { perm. }
\end{aligned}
$$

In the flattened configuration, $k_{1}=k_{2}=k_{3}=k_{4} / 3$, I again find that only the terms where $\mathcal{R}_{k_{4}}$ is in the innermost commutator grow as $\alpha^{6}$. Among the 9 remaining permutations there are 2 distinct scalar products in $\mathcal{K}_{2}: \mathbf{k}_{1} \cdot\left(-\mathbf{k}_{12}\right)=-2 k^{2}+5$ perm. , $\mathbf{k}_{1} \cdot \mathbf{k}_{2}=k^{2}+2$ perm. In the case of $\mathcal{K}_{3}$ there are 3 distinct cases: $\mathbf{k}_{1} \cdot\left(-\mathbf{k}_{14}\right)=2 k^{2}, \mathbf{k}_{1} \cdot \mathbf{k}_{4}=-3 k^{2}, \mathbf{k}_{4} \cdot\left(-\mathbf{k}_{14}\right)=-6 k^{2}$, each with 3 permutations $k_{1} \rightarrow\left\{k_{2}, k_{3}\right\}$. After taking that into account one finds

$$
\begin{aligned}
& \mathcal{K}_{2}^{\text {flat }}(k, k, k, 3 k)=-\frac{80}{81}\left(\frac{A}{\left|c_{s}^{2}\right|}\right) \alpha^{6}\left|c_{s}\right|^{4} k^{3}, \\
& \mathcal{K}_{3}^{\text {flat }}(k, k, k, 3 k)=-\frac{176}{81}\left(\frac{A}{\left|c_{s}^{2}\right|}\right) \alpha^{6}\left|c_{s}\right|^{4} k^{3} .
\end{aligned}
$$



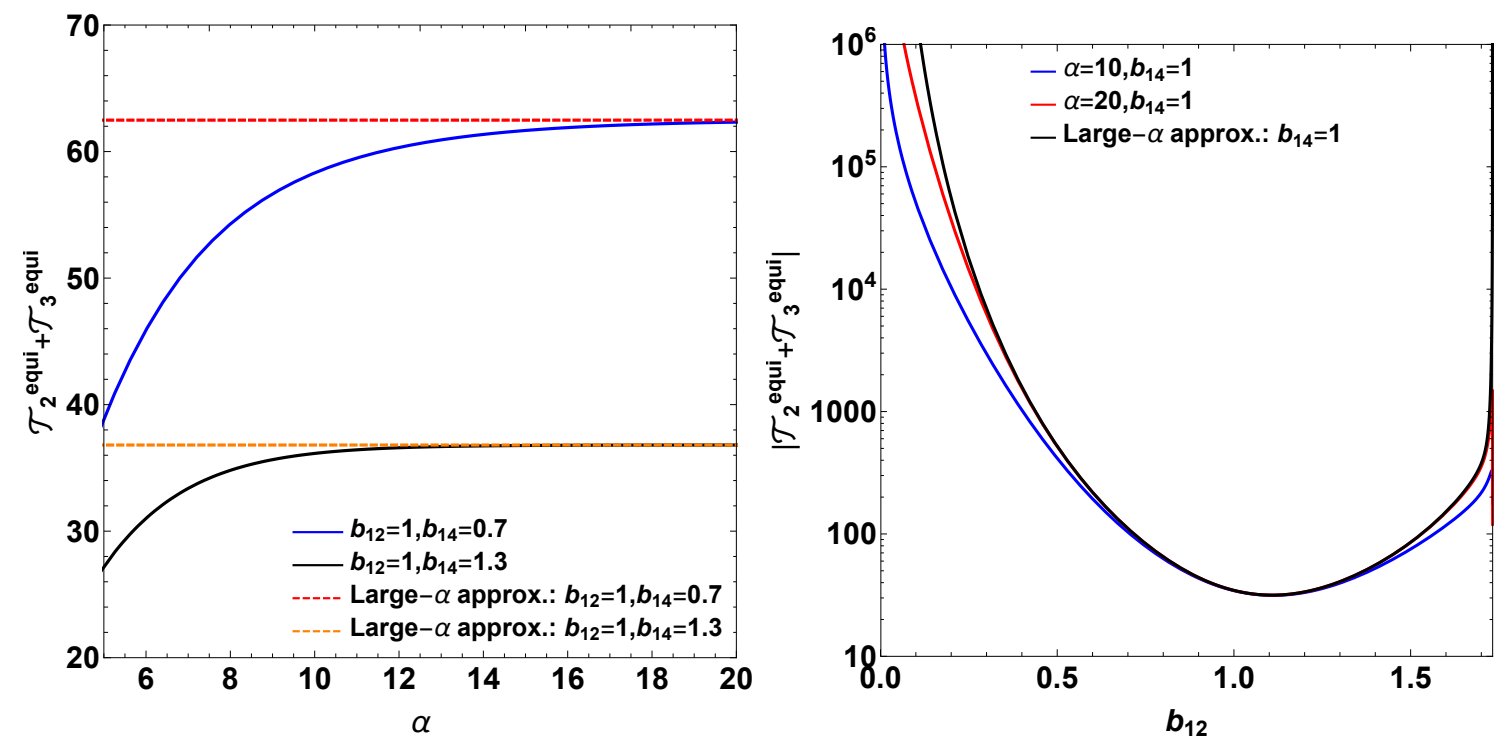

Figure 5: Equilateral trispectrum, $\mathcal{T}_{2}^{\text {equi }}+\mathcal{T}_{3}^{\text {equi }}$, as a function of: (left plot) $\alpha$ for 2 different values of $b_{14}$, (right plot) $b_{12}$ for two different values of $\alpha$. I also compare the full expressions with the large- $\alpha$ approximation. I fixed $\left|c_{s}^{2}\right|=1, A=1$ in both plots.

The large- $\alpha$ approximation is again accurate and yields the trispectrum

$$
\begin{aligned}
\mathcal{T}_{\text {cross }}^{\text {flat }}(k, k, k, 3 k) & =-\frac{1}{2^{9} k^{3} 3^{3 / 4}\left|c_{s}\right|^{2}}\left(\frac{1}{\left|c_{s}^{2}\right|}+1\right)^{2}\left(\mathcal{K}_{2}^{\text {flat }}(k, k, k, 3 k)+\mathcal{K}_{3}^{\text {flat }}(k, k, k, 3 k)\right) \\
& \simeq \frac{A \alpha^{6}}{162 \cdot 3^{3 / 4}}\left(\frac{1}{\left|c_{s}^{2}\right|}+1\right)^{2} .
\end{aligned}
$$

In the equilateral limit, $k_{1}=k_{2}=k_{3}=k_{4}=k$, among the 18 permutations 12 involve scalar products of $\mathbf{k}_{i} \cdot\left(-\mathbf{k}_{i j}\right)$ while the other 6 involve terms of the form $\mathbf{k}_{i} \cdot \mathbf{k}_{j}$. Nevertheless, because of momentum conservation, there are only 3 different angles. Therefore, the terms in $\mathbf{k}_{i} \cdot\left(-\mathbf{k}_{i j}\right)$ repeat 4 times while the ones on $\mathbf{k}_{i} \cdot \mathbf{k}_{j}$ repeat twice. The final expressions in the large- $\alpha$ limit are not very illuminating so I present them in eqs. (C.5) and (C.6) of the appendix. In fig. 5 I plot $\mathcal{T}_{2}^{\text {equi }}+\mathcal{T}_{3}^{\text {equi }}$ and compare it with the large- $\alpha$ approximations. Similarly to $\mathcal{K}_{1}^{\text {equi }}$, in the collinear limit, $b_{12} \rightarrow\{0,2\}$, the trispectrum grows with $\alpha$.

Terms in $\mathcal{K}_{4}$ : Finally I evaluate the terms in $\mathcal{K}_{4}$. The expression for the integral in eq. (3.45) after inserting the mode functions is long so I give it in eq. (C.8) and only discuss here the flattened and equilateral shapes. In the flattened case, $k_{1}=k_{2}=k_{3}=k_{4} / 3$, among the leading 27 terms where $k_{4}$ is in the innermost commutator there are 6 different combinations:

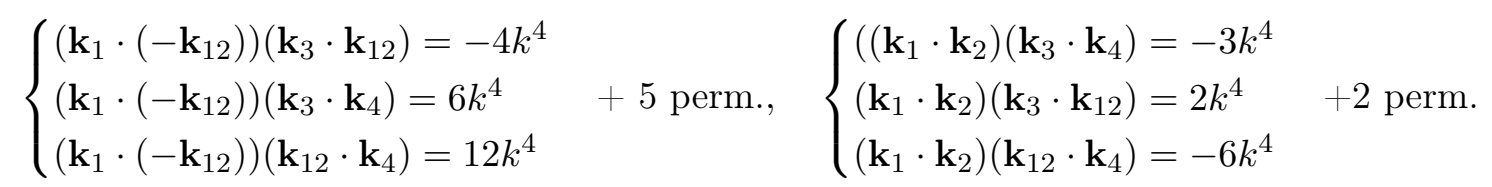

After summing the different contributions, the trispectrum in the flattened limit reads

$$
\mathcal{T}_{4}^{\text {flat }}(k, k, k, 3 k)=\frac{55}{7776 \cdot 3^{3 / 4}} \alpha^{6}\left(\frac{1}{\left|c_{s}^{2}\right|}+1\right)^{2} .
$$



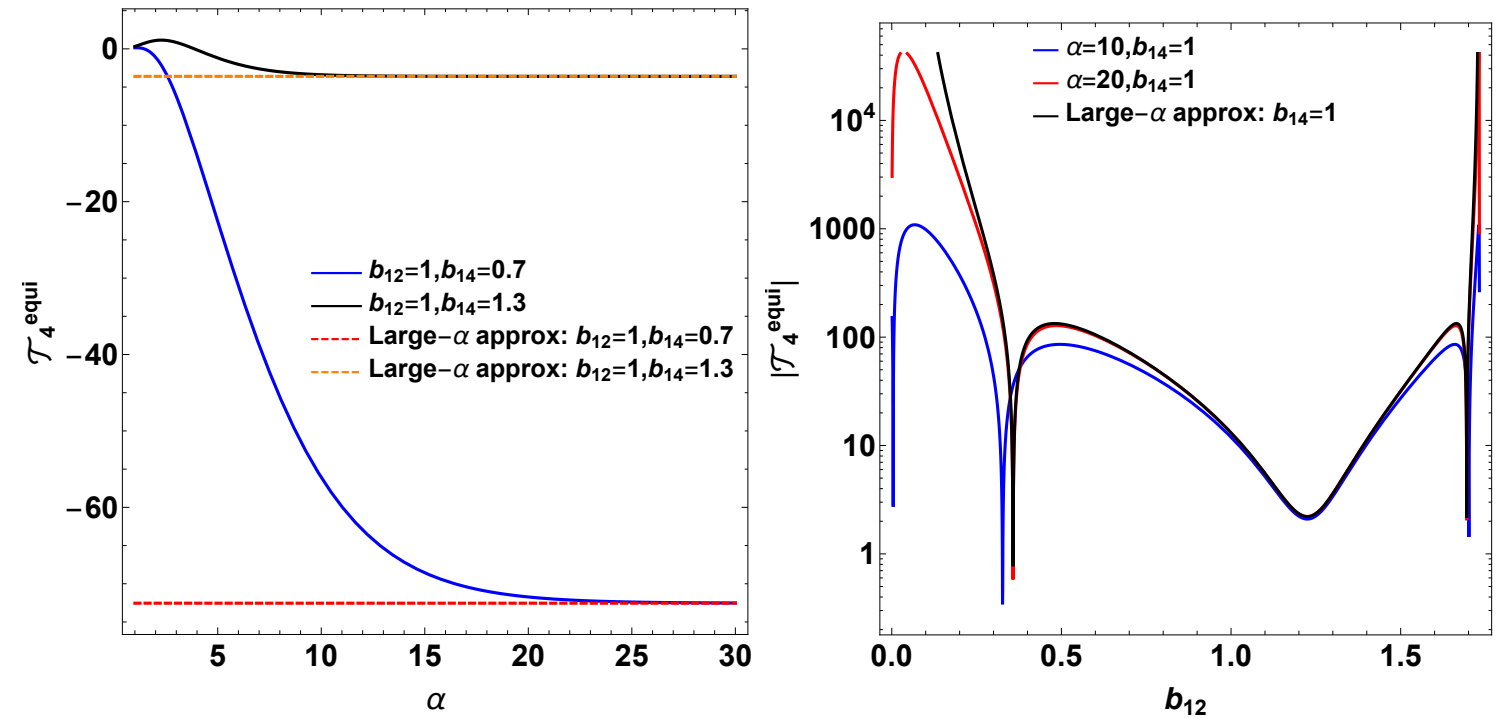

Figure 6: Equilateral trispectrum, $\mathcal{T}_{4}^{\text {equi }}$, as a function of: (left plot) $\alpha$ for 2 different values of $b_{14}$, (right plot) $b_{12}$ for two different values of $\alpha$. I also compare the full expressions with the large- $\alpha$ approximation. I fixed $\left|c_{s}^{2}\right|=1, A=1$ in both plots.

In the equilateral case, $k_{1}=k_{2}=k_{3}=k_{4}$, there are 12 different combinations corresponding to:

$$
\left\{\begin{array}{l}
2\left(\mathbf{k}_{1} \cdot \mathbf{k}_{2}\right)\left(\mathbf{k}_{3} \cdot \mathbf{k}_{4}\right)=2 k^{4} \cos \left(\theta_{12}\right)^{2} \\
4\left(\mathbf{k}_{1} \cdot\left(-\mathbf{k}_{12}\right)\right)\left(\mathbf{k}_{3} \cdot \mathbf{k}_{4}\right)=-4 k^{4} \cos \left(\theta_{12}\right)\left(1+\cos \left(\theta_{12}\right)\right) \\
4\left(\mathbf{k}_{1} \cdot \mathbf{k}_{2}\right)\left(\mathbf{k}_{3} \cdot \mathbf{k}_{12}\right)=-4 k^{4} \cos \left(\theta_{12}\right)\left(1+\cos \left(\theta_{12}\right)\right) \\
8\left(\mathbf{k}_{1} \cdot\left(-\mathbf{k}_{12}\right)\right)\left(\mathbf{k}_{3} \cdot \mathbf{k}_{12}\right)=8 k^{4}\left(1+\cos \left(\theta_{12}\right)\right)\left(1+\cos \left(\theta_{12}\right)\right)
\end{array}+2\right. \text { perm }
$$

I give the full expression for the trispectrum in the equilateral shape, $\mathcal{T}_{4}^{\text {equi }}$, in eq. (C.8) and plot it in fig. 6. The behavior is qualitatively similar to that of $\mathcal{T}_{1,2,3}^{\text {equi }}$.

To summarize, all contributions of the exchange diagram to the trispectrum peak on certain collapsed shapes, the leading ones proportionally to $\alpha^{6}$, while other configurations give an $\alpha$-independent contribution in the large- $\alpha$ limit.

\section{Observational constraints}

After having collected and derived a few predictions for the spectrum of scalar perturbations, I can now use the latest observational data to constraint the models described by the EFT with imaginary sound speed, i.e. models where the entropic directions have a large and negative effective mass.

The parameter $x$, appearing in the mode functions in eq. (2.5), controls the amplitude and tilt of the power spectrum, while $\alpha$ controls the EFT cutoff and the size of the nonGaussianities. Although these two parameters are related they might not be equal as it is clear from the case of rapid-turn attractors where $x=\alpha \pi\left(\sqrt{1+\left|c_{s}\right|^{2}}+1\right) /\left(2\left|c_{s}\right|\right)$ (see app. A). Therefore, constraints on the power spectrum only restrict $x$ while non-Gaussian constraints only affect $\alpha$. 


\subsection{Power spectrum and spectral index}

The amplitude and tilt of the scalar power spectrum at CMB scales are measured to be $P_{\zeta}^{\text {obs }}=2.2 \times 10^{-9}, n_{s}^{\text {obs }}=0.965$ [4]. On small scales, the spectrum is further constrained by the overproduction of primordial black holes $(\mathrm{PBH})[38]$

$$
P_{\zeta} \lesssim P_{\zeta}^{\mathrm{PBH}} \sim 10^{-3}-10^{-2}
$$

In the EFT described in sec. 2 the scalar power spectrum for $x \gg 1$ is approximately given by eqs. (2.7) and $(2.8)[11,14]$. If the $k$-dependence of $\rho$ and $\theta$ is much milder than that of $e^{2 x}$, as it tends to be the case, then the spectral tilt is essentially controlled by $x$, namely,

$$
n_{s}-1 \simeq-2 \epsilon-\delta+2 \frac{\dot{x}}{H}
$$

where $\delta \equiv \dot{\epsilon} /(\epsilon H)$. The PBH constraint is quite constraining if $x$ is proportional to some positive power of the first slow-roll parameter, $x=x_{f} \epsilon^{n}$. In that case, $x$ grows in time and exponentially enhances $P_{\zeta}$ on small scales. Using the fact that $\epsilon \simeq 1$ at the end of inflation, and assuming that $\rho, \theta$ do not vary much during inflation, eq. (4.1) then implies that

$$
P_{\zeta}^{\mathrm{obs}} \epsilon_{\mathrm{CMB}} \exp \left[2\left(1-\epsilon_{\mathrm{CMB}}^{n}\right) x_{f}\right] \lesssim P_{\zeta}^{\mathrm{PBH}}
$$

Moreover, in such cases the spectral index is given, for $x \gg 1$, by

$$
n_{s}-1 \simeq-2 \epsilon+2 n x \delta \text {. }
$$

This requirement jointly with eq. (4.3) strongly constraints the background dynamics. For example, in the case of hyperinflation [11] where $n=1 / 2$, eq. (4.3) translates, using eqs. (A.2) and (A.5), into a constraint on the field-space curvature $L$

$$
\frac{M_{p}}{L} \lesssim \frac{1}{(2-\sqrt{2}) \sqrt{2} \pi\left(1-\sqrt{\epsilon_{\mathrm{CMB}}}\right)} \log \left(\frac{P_{\zeta}^{\mathrm{PBH}}}{\epsilon_{\mathrm{CMB}} P_{\zeta}^{\mathrm{obs}}}\right) .
$$

More generally, if $x \propto \epsilon^{n}$ then from eqs. (4.3) and (4.4) models such that at CMB scales:

1. $\epsilon \lesssim-x \delta$ (e.g. polynomial potentials) are excluded as they lead to a blue scalar spectrum.

2. $\epsilon \simeq 0.02 \gg 2 n x \delta$ (e.g. exponential potentials) are generically outside the region of validity, $x \gg 1$, unless $n \ll 1$, because $x \lesssim 8.5 \times 0.02^{n}$.

3. $\epsilon \ll 1, n x \delta \simeq \mathcal{O}(-0.01)$ (e.g. hilltop-like potentials) are still viable. In this case, by fine-tuning the initial conditions it is possible to satisfy all the constraints.

\subsection{Non-Gaussianities}

In this section, I translate the observational constraints on the bispectrum and trispectrum into constraints on $\alpha$. The constraints apply to all models described by the EFT in sec. 2 . How those constraints affect the parameters of the UV model will then vary in each case. In the end I particularize to the case rapid-turn attractors described in app. A [11, 19]. Note that the results for the bi and trispectrum in the collapsed limits should only be trusted at the order of magnitude level because of their UV sensitivity, as discussed in the beginning of sec. 3 . 


\section{Bispectrum}

I start by the bispectrum where a detailed study has been performed in [30, 32]. Here I review those results and make a direct comparison with the observational constraints to get a constraint $\alpha$.

The bispectrum constraints are given in terms of $B_{\Phi}\left(k_{1}, k_{2}, k_{3}\right)=(3 / 5)^{3} B\left(k_{1}, k_{2}, k_{3}\right)$ where $\Phi=3 / 5 \zeta$ is the Newtonian potential. Two characteristic templates are the equilateral and orthogonal respectively given by

$$
\begin{aligned}
& B_{\Phi}^{\text {equil }}\left(k_{1}, k_{2}, k_{3}\right)= \\
& \quad 6 \Delta_{\Phi}^{2} f_{\mathrm{NL}}^{\text {equi }}\left[-\frac{1}{\left(k_{1} k_{2}\right)^{3}}-\frac{1}{\left(k_{2} k_{3}\right)^{3}}-\frac{1}{\left(k_{3} k_{1}\right)^{3}}-\frac{2}{\left(k_{1} k_{2} k_{3}\right)^{2}}+\left[\frac{1}{k_{1} k_{2}^{2} k_{3}^{3}}+5 \text { perms. }\right]\right], \\
& B_{\Phi}^{\text {orth }}\left(k_{1}, k_{2}, k_{3}\right)= \\
& \quad 6 \Delta_{\Phi}^{2} f_{\mathrm{NL}}^{\text {orth }}\left[-\frac{3}{\left(k_{1} k_{2}\right)^{3}}-\frac{3}{\left(k_{2} k_{3}\right)^{3}}-\frac{3}{\left(k_{3} k_{1}\right)^{3}}-\frac{8}{\left(k_{1} k_{2} k_{3}\right)^{2}}+\left[\frac{3}{k_{1} k_{2}^{2} k_{3}^{3}}+5 \text { perms. }\right]\right]
\end{aligned}
$$

where $\Delta_{\Phi}=(3 \sqrt{2} \pi / 5)^{2} P_{\zeta}$. When confronting the predictions with the observational data, optimally, one should construct a template dedicated to the scenario under scrutinity. However, as it was shown in $[30,39]$ the orthogonal template is strongly correlated with the bispectrum generated in this model for large $\alpha$. Therefore, I can use the observational constraint $f_{\mathrm{NL}}^{\text {orth }}=-38 \pm 24(68 \% \mathrm{CL})[40]$ to place constraints on $\alpha$. More concretely, using eqs. (3.4), (3.10) and (3.16) and the fact that in the flattened limit $B_{\Phi}^{\text {orth }}(2 k, k, k)=-3 \Delta_{\Phi}^{2} f_{\mathrm{NL}}^{\text {ortho }} / k^{6}$ one finds that

$$
f_{N L}^{\text {ortho }} \simeq-\frac{5}{288}(A+1)\left(\frac{1}{\left|c_{s}^{2}\right|}+1\right) \alpha^{3} .
$$

The parameters $A$ and $c_{s}$ depend on the microphysics of each model. However, unless $A$ is close to -1 , the absolute value of $f_{\mathrm{NL}}$ is roughly bounded from below and essentially depends on $\alpha$. Thus, imposing the $2 \sigma$ constraint $-86<f_{N L}^{\text {ortho }}<10[40]$ one finds

$$
-8 \lesssim \alpha(A+1)^{1 / 3}\left(\frac{1}{\left|c_{s}^{2}\right|}+1\right)^{1 / 3} \lesssim 17
$$

For rapid-turn models, where $\alpha^{2}=4\left|c_{s}\right|^{2} /\left(1+\left|c_{s}\right|^{2}\right) \omega^{2}$ and $\omega$ is the turning rate in field space defined in eq. (A.1), it implies that

$$
-4 \lesssim \omega\left|c_{s}\right|(A+1)^{1 / 3}\left(\frac{1}{\left|c_{s}^{2}\right|}+1\right)^{-1 / 6} \lesssim 9
$$

which, in the particular case of hyperinflation $\left(A \simeq-1 / 3,\left|c_{s}^{2}\right|=1\right)[17,32]$, imposes $\omega \lesssim 11$.

\section{Trispectrum}

In sec. 3.2 I derived the contributions to the trispectrum from the contact interaction and the exchange diagram in fig. 2. I derived the general expressions and then focused on two particular shapes: the equilateral, $k_{1}=k_{2}=k_{3}=k_{4}=k$, and the flattened, $k_{1}=k_{2}=$ $k_{3}=k_{4} / 3=k$. In this section, I focus on the flattened as it the representative of the leading contributions, and derive consequent constraints on $\alpha$. 
From the contact term eqs. (3.28), (3.33) and (3.37) give the trispectrum

$$
\mathcal{T}_{\text {contact }}^{\text {flat }}=\frac{\alpha^{5}}{180 \cdot 3^{3 / 4}}\left(\frac{1}{\left|c_{s}^{2}\right|}+1\right)\left(B\left|c_{s}\right|^{2}-C+\frac{1}{4}\right),
$$

while from the exchange diagram eqs. (3.49), (3.57) and (3.58) yield

$$
\mathcal{T}_{\text {exchange }}^{\text {flat }}=\frac{\alpha^{6}}{162 \cdot 3^{3 / 4}}\left(\frac{1}{\left|c_{s}^{2}\right|}+1\right)\left(\frac{3}{16} A^{2}+A+\frac{55}{48}\right) .
$$

The current observational constraints are given in terms of parameters associated with local and contact interactions. At $68 \%$ CL they $\operatorname{read}^{6}[40]$

$$
\begin{gathered}
g_{\mathrm{NL}}^{\text {local }}=(-5.8 \pm 6.5) \times 10^{4}, \quad g_{\mathrm{NL}}^{\dot{\sigma}^{4}}=(-0.8 \pm 1.9) \times 10^{6}, \\
g_{\mathrm{NL}}^{(\partial \sigma)^{4}}=(-3.9 \pm 3.9) \times 10^{5} .
\end{gathered}
$$

Contributions to local non-Gaussianities are typically small in these models due to the decay of the isocurvature mode on superhorizon scales. Therefore, I disregard $g_{\mathrm{NL}}^{\text {local }}$ and focus instead on $g_{\mathrm{NL}}^{\dot{\sigma}^{4}}$ and $g_{\mathrm{NL}}^{(\partial \sigma)^{4}}$ which are defined through [41]

$$
\begin{aligned}
\left\langle\zeta_{k_{1}} \zeta_{k_{2}} \zeta_{k_{3}} \zeta_{k_{2}}\right\rangle^{\prime}= & (2 \pi)^{3} \frac{221184}{25} g_{\mathrm{NL}}^{\dot{\sigma}^{4}} P_{\zeta}^{3} \frac{1}{k_{1} k_{2} k_{3} k_{4} K^{5}} \\
\left\langle\zeta_{k_{1}} \zeta_{k_{2}} \zeta_{k_{3}} \zeta_{k_{2}}\right\rangle^{\prime}= & (2 \pi)^{3} \frac{165888}{2575} g_{\mathrm{NL}}^{(\partial \sigma)^{4}} P_{\zeta}^{3}\left(\frac{2 K^{4}-2 K^{2} \Sigma k_{i}^{2}+K \sum k_{i}^{3}+12 k_{1} k_{2} k_{3} k_{4}}{k_{1}^{3} k_{2}^{3} k_{3}^{3} k_{4}^{3} K^{5}}\right) \times \\
& {\left[\left(\mathbf{k}_{1} \cdot \mathbf{k}_{2}\right)\left(\mathbf{k}_{3} \cdot \mathbf{k}_{4}\right)+2 \text { perm. }\right] }
\end{aligned}
$$

where $K=\sum_{i} k_{i}$ and the prime in the correlator denotes that the delta function was suppressed $^{7}$. Unfortunately, these templates are not optimized for contributions from exchanged diagrams which tend to be subdominant, contrarily to what happens in the models studied here. In light of that, I will first constraint $\alpha$ using the contribution to the trispectrum from the contact interaction in eq. (4.13). This contribution should have a larger correlation with the templates associated with $g_{\mathrm{NL}}^{\dot{\sigma}^{4}}, g_{\mathrm{NL}}^{(\partial \sigma)^{4}}$. Afterwards, I use the same constraint on $g_{\mathrm{NL}}^{\dot{\sigma}^{4}}$ as a proxy to constraint the exchange diagram. Namely, by imposing the $2 \sigma$ constraint on $g_{\mathrm{NL}}^{\dot{\sigma}^{4}}$ in eq. (4.15) as a benchmark ${ }^{8}$ I find, using eq. (4.13), that

$$
\frac{675}{275 \cdot 3^{9 / 4}}\left|\mathcal{T}^{\text {near-flat }}\right| \lesssim 5 \times 10^{6} \Rightarrow \alpha \lesssim 98\left(B\left|c_{s}\right|^{2}-C+\frac{1}{4}\right)^{-1 / 5}
$$

Repeating the same steps for the contribution from the exchange diagram in eq. (4.14), bearing in mind the caveats mentioned above, yields

$$
\alpha \lesssim 34.4\left[\left(\frac{1}{\left|c_{s}^{2}\right|}+1\right)\left(\frac{3}{16} A^{2}+A+\frac{55}{48}\right)\right]^{-1 / 6} .
$$

By comparing eqs. (4.19), (4.20) with eq. (4.11), I conclude that for $A, B, C, c_{s} \sim \mathcal{O}(1)$ the bispectrum currently provides the strongest constraints.

\footnotetext{
${ }^{6}$ For $g_{\mathrm{NL}}^{\dot{\sigma}^{4}}, g_{\mathrm{NL}}^{(\partial \sigma)^{4}}$ I used the constraint in the first line of table 24 of [40].

${ }^{7}$ Note that I use a different Fourier convention and so the correlator differs by a factor of $(2 \pi)^{3}$ compared to the notation in [40].

${ }^{8}$ Using instead the constraint on $g_{\mathrm{NL}}^{(\partial \sigma)^{4}}$ gives similar results.
} 


\section{Conclusion}

In this work, I studied inflationary models described by a single-field EFT with an imaginary speed of sound [14]. Such EFT arises when the effective entropic mass(es), $m_{s}$, are large and negative. Models where the field-space metric is hyperbolic and a large turning rate in fieldspace counterbalances the instability are among the interesting examples [10-21]. Slow-roll inflation is then still possible, even in steeper potentials, albeit in a different attractor. The spectrum of scalar perturbations also has interesting new features. Its amplitude is enhanced by $\sim e^{2 x}$ where $x$ is closely related to $m_{s} / H[14,30]$. Interestingly, non-Gaussian parameters only have a polynomial dependence on $m_{s} / H$ [30-32].

In sec. 3 I extended the discussion of non-Gaussianities in these models. First, I reviewed the bispectrum calculation in sec. 3.1. Then, in sec. $3.2 \mathrm{I}$ completed the trispectrum calculation by computing contributions from both the contact interaction and the exchange diagram in fig. 2. So far only one term had been computed [32]. I found that for most shapes the bi and trispectrum are constant in the limit $\alpha=\left|c_{s} m_{s}\right| / H \gg 1$. However, for some configurations where all momenta collapse to a line (e.g. the flattened shapes in fig. 1) the time integrals become dominated by the UV cutoff and proportional to $\alpha^{3}$ in the bispectrum (eqs. (3.10), (3.16)). In the trispectrum, the dominant contribution, proportional to $\alpha^{6}$ (eq. (4.14)), comes from the exchange diagram while the contact interaction gives a contribution proportional to $\alpha^{5}$ (eq. (4.13)), as anticipated in [32]. When overlapping, the results found in this work agree quantitatively and qualitatively with the literature. Only the contribution to the trispectrum in eq. (3.49) differs a factor of 3 from the result found in [32]. The UV sensitivity for the flattened shapes is typical of theories with excited initial states [33, 34] and implies that a precise calculation of non-Gaussianities is only achievable in the full multi-field system. Nevertheless, as I argued in sec. 3, if the fields quickly approach the massless and weakly coupled limit for scales above the cutoff, which is typically the case, they will be described by plane waves whose rapid oscillations exponentially suppress the contribution above the cutoff. Therefore, the results for the flattened shapes within the EFT still provide good order of magnitude estimates.

Finally, in sec. 4 I confronted the different predictions for the spectrum of scalar perturbations against observations. I pointed out that if $x$ is proportional to some positive power of the slow-roll parameter $\epsilon$, as in the case of the rapid-turn attractors like hyperinflation [11] or sidetracked inflation [14], the spectrum grows exponentially on small scales and so might overproduce primordial black holes. This provides a strong constraint for scenarios where the spectral index is controlled by $\epsilon$. For example, for hyperinflation, the combination of a red spectral tilt at CMB scales with the PBH constraint excludes exponential and polynomial-like potentials.

I then used the observational constraints on non-Gaussianities [40] to constrain the EFT parameter $\alpha$. For the bispectrum, I used the constraint on $f_{\mathrm{NL}}^{\text {ortho }}$ whose template has been shown to strongly correlate with the bispectrum in this model [30, 42]. I found in eq. (4.11) the constraint on $\alpha$ and in eq. (4.12) I translated it in terms of the parameter $\omega$ which controls the turning-rate in field space in rapid-turn attractors. For hyperinflation, I found that $\omega \lesssim 11$ which strongly constraints the model. Regarding the trispectrum, I used the constraints on the parameters $g_{\mathrm{NL}}^{\dot{\sigma}^{4}}, g_{\mathrm{NL}}^{(\partial \sigma)^{4}}[40]$ to constraint the contribution generated from the contact interaction, eq. (4.19), and from the exchange diagram, eq. (4.20). For the exchange diagram, which is the dominant contribution for large $\alpha$, a dedicated analysis is required as none of the associated templates is expected to be a good fit. Still, I used 
the same constraints as a proxy. In both cases, I found that the constraints on $\alpha$ from the trispectrum are weaker than that coming from the bispectrum. However, a dedicated analysis is required to make more definite statements.

To conclude, I derived different constraints on the models described by the EFT with imaginary sound speed. Generically, I found that although constrained these models are still observationally viable if the parameter controlling the effective mass is roughly in the window $\alpha \lesssim 10-20$. Furthermore, if the parameter $x$ is proportional to a positive power of $\epsilon$ the only viable models are likely those where the spectral tilt is controlled by $2 \dot{x} / H \simeq \mathcal{O}(-0.01)$, like in hill-top potentials.

\section{Acknowledgments}

I would like to thank David M.C. Marsh and Theodor Bjorkmo for several discussions regarding hyperinflation and rapid-turn attractors and, in particular, David M.C. Marsh for discussions related to the trispectrum computation. I would also like to thank Sebastien Renaux-Petel and Jacopo Fumagali for comments on a draft of this paper.

\section{A Rapid-turn attractors}

A negatively curved field-space metric can potentially destabilize the inflationary trajectory [10-21]. This instability can, however, be counterbalanced by a large angular velocity in fieldspace thus allowing for inflation in a new attractor solution. Recently, it has been argued that all such models follow the same rapid-turn attractor where the turning rate in field-space is characterized by [19]

$$
\omega=\left\|\mathcal{D}_{t}\left(\dot{\phi}^{a} / \dot{\phi}\right)\right\| / H .
$$

The superscript $a$ runs over the $N$-dimensional field-space, $\dot{\phi}=\left\|\dot{\phi}^{a}\right\|$ and $\mathcal{D}_{t}$ is a covariant time derivative in the field-space metric. The large turning rate effectively generates a large and negative mass, $m_{s}^{2}=(\xi-1) \omega^{2} H^{2}$, for the entropic direction. This direction can then be integrated out leading to the single-field EFT described in sec. 2 with [31]

$$
x \simeq(2-\sqrt{3+\xi}) \pi \omega / 2, \quad\left|c_{s}\right|=\sqrt{(1-\xi) /(3+\xi)},
$$

where $\xi<1$. Below I briefly present two examples of such attractors with hyperbolic geometries: hyperinflation [11] and sidetracked inflation [14].

- Hyperinflation: It is described by the Lagrangian [11]

$$
\mathcal{L}=-\frac{1}{2}(\partial \phi)^{2}-\frac{1}{2} \sinh ^{2}(\phi / L)(\partial \psi)^{2}-V(\phi),
$$

The new attractor solution is characterized by

$$
\dot{\phi}=-3 H L, \quad \dot{\psi} \sinh \frac{\phi}{L}=\sqrt{L \partial_{\phi} V-(3 H L)^{2}} \equiv \omega L H
$$

with slow parameters given in the large $\omega$ limit by

$$
\epsilon=-\frac{\dot{H}}{H^{2}} \simeq \frac{1}{2} \omega^{2}\left(\frac{L}{M_{p}}\right)^{2}, \quad \eta=\frac{L V_{, \phi \phi}}{V_{, \phi}} .
$$


with $\omega L \ll M_{p}$. It is then clear that inflation can unfold in steeper potentials. The effective entropic mass is in this case $m_{s}^{2}=-2 \omega^{2} H^{2}$. Therefore, for $\omega \gg 1$ the perturbations can be described by the EFT of sec. 2. The power spectrum is given by eq. (4.2) with $2 \rho \sin \theta=1$ while the spectral tilt is given by [12]

$$
n_{s}-1=-2 \epsilon+x \eta
$$

- Sidetracked inflation: The system is characterized by the Lagrangian [14]

$$
\mathcal{L}=-\frac{1}{2}\left(1+\frac{2 \psi^{2}}{M^{2}}\right)(\partial \phi)^{2}-\sqrt{2} \frac{\psi}{M} \partial \phi \partial \psi-(\partial \psi)^{2}-V(\phi, \psi)
$$

where $V(\phi, \psi)=V_{1}(\phi)+m^{2} \psi^{2} / 2$ and $m \gg H$. In the attractor solution $\psi \sim H$ and the entropic mass is

$$
\frac{m_{s}^{2}}{H^{2}} \simeq 12 \frac{m}{H} \frac{\psi^{2}}{M^{2}} \operatorname{sign}\left(V_{, \varphi}\right) .
$$

Therefore, if $V^{\prime}<0$ then $\left|m_{s}\right| / H \gg 1$ and the theory can be described by the EFT with imaginary sound speed given by $c_{s}^{2} \simeq 3 H /(2 m) \operatorname{sign}\left(V_{, \varphi}\right)$.

\section{B Interaction Hamiltonians}

As we discussed in the previous sections the system under discussion can be well described by a single field EFT. Therefore, to leading order in the slow-roll parameters the system will inherit the interactions derived in the context of the EFT of single-field inflation $[23,30]$.

\section{B.1 Cubic order}

The interaction Hamiltonian of cubic order to leading order in slow-roll and derivatives is given by [23]

$$
H_{I, 3}(\tau)=-\int d^{3} x \frac{a \epsilon M_{\mathrm{Pl}}^{2}}{H}\left(\frac{1}{c_{s}^{2}}-1\right)\left[\zeta^{\prime}(\vec{\nabla} \zeta)^{2}+\frac{A}{c_{s}^{2}} \zeta^{\prime 3}\right]
$$

For two-field models within the validity of the EFT, $A$ is given by [17]

$$
A=-\frac{1}{2}\left(1+c_{s}^{2}\right)+\frac{2}{3}\left(1+2 c_{s}^{2}\right) \frac{\epsilon H^{2} M_{p}^{2} R_{\mathrm{fs}}}{m_{s}^{2}}-\frac{1}{6}\left(1-c_{s}^{2}\right)\left(\frac{\kappa V_{; s s s}}{m_{s}^{2}}+\frac{\kappa \epsilon H^{2} M_{\mathrm{Pl}}^{2} R_{\mathrm{fs}, s}}{m_{s}^{2}}\right)
$$

where $\kappa=\sqrt{2 \epsilon} M_{p} / \eta_{\perp}$. For hyperinflation $A \simeq-1 / 3$ [17]. After Fourier transforming the fields according to

$$
\zeta(x)=\int \frac{d^{3} k}{(2 \pi)^{3 / 2}} e^{i k x}\left[a_{k} \zeta_{k}+a_{-k}^{\dagger} \zeta_{k}^{*}\right], \quad\left[a_{k}, a_{q}^{\dagger}\right]=\delta^{(3)}(k-q),
$$

where $a, a^{\dagger}$ are the creation and annihilation operators and using $a \simeq-1 /(\tau H), P_{\zeta, 0}=$ $H^{2} /\left(8 \pi^{2}\left|c_{s}\right| \epsilon M_{p}^{2}\right)$ and eq. (2.10), the interaction Hamiltonian becomes of the form

$$
H_{I, 3}(\tau)=h\left(\tilde{H}_{3,1}+\tilde{H}_{3,2}\right),
$$


where

$$
\begin{aligned}
h & \equiv\left(\frac{1}{\left|c_{s}^{2}\right|}+1\right) \frac{1}{8\left|c_{s}\right|} \sqrt{\frac{P_{\zeta, 0}}{8 \pi}}, \\
\tilde{H}_{3,1} & =\frac{\left(\mathbf{k}_{1} \cdot \mathbf{k}_{2}\right)}{\tau} \int\left(\prod_{i=1}^{3} d^{3} k_{i}\right) \delta^{(3)}\left(k_{1}+k_{2}+k_{3}\right) \mathcal{R}_{k_{1}} \mathcal{R}_{k_{2}} \mathcal{R}_{k_{3}}^{\prime}, \\
\tilde{H}_{3,2} & =\frac{A}{\tau\left|c_{s}^{2}\right|} \int\left(\prod_{i=1}^{3} d^{3} k_{i}\right) \delta^{(3)}\left(k_{1}+k_{2}+k_{3}\right) \mathcal{R}_{k_{1}}^{\prime} \mathcal{R}_{k_{2}}^{\prime} \mathcal{R}_{k_{3}}^{\prime},
\end{aligned}
$$

and I used the fact that $c_{s}^{2}<0$.

\section{B.2 Quartic order}

Up to 4-derivatives and to lowest order in the slow-roll parameters the interaction Hamiltonian to fourth order has the form $[34]^{9}$

$$
H_{I, 4}(\tau)=\int \frac{d^{3} x}{H^{4}}\left[\left(-\mu+9 \frac{\lambda^{2}}{\Sigma}\right) \zeta^{\prime 4}+\left(3 \lambda c_{s}^{2}-\Sigma\left(1-c_{s}^{2}\right)\right)\left(\partial \zeta_{I}\right)^{2} \zeta^{\prime 2}-\frac{c_{s}^{2}}{4} \Sigma\left(c_{s}^{2}-1\right)(\partial \zeta)^{4}\right]
$$

where $\Sigma=M_{p}^{2} H^{2} \epsilon / c_{s}^{2}$ [43]. The terms in $\lambda$ and $\mu$ need to be derived from the full multi-field system as they cannot be determined uniquely from background quantities and the speed of sound. I leave the coefficients of those terms $B, C$ undetermined and define the Hamiltonian as

$$
H_{I, 4}(\tau)=\int d^{3} x\left(\frac{1}{c_{s}^{2}}-1\right) \frac{\epsilon M_{p}^{2}}{H^{2}}\left[B \zeta^{\prime 4}+C(\partial \zeta)^{2} \zeta^{\prime 2}-\frac{c_{s}^{2}}{4}(\partial \zeta)^{4}\right]
$$

In Fourier space, and in terms of $\mathcal{R}$ defined in eq. (2.10), the Hamiltonian becomes

$$
\begin{aligned}
& H_{I, 4}(\tau)=\left(\frac{1}{c_{s}^{2}}-1\right) \frac{P_{\zeta, 0}}{32(2 \pi)\left|c_{s}\right|} \int\left(\prod_{i=1}^{4} d^{3} k_{i}\right) \delta^{(3)}\left(\sum_{i=1}^{4} k_{i}\right) \times \\
& \times\left[B \mathcal{R}_{k_{1}}^{\prime} \mathcal{R}_{k_{2}}^{\prime} \mathcal{R}_{k_{3}}^{\prime} \mathcal{R}_{k_{4}}^{\prime}-C\left(\mathbf{k}_{1} \cdot \mathbf{k}_{2}\right) \mathcal{R}_{k_{1}} \mathcal{R}_{k_{2}} \mathcal{R}_{k_{3}}^{\prime} \mathcal{R}_{k_{4}}^{\prime}-\frac{c_{s}^{2}}{4}\left(\mathbf{k}_{1} \cdot \mathbf{k}_{2}\right)\left(\mathbf{k}_{3} \cdot \mathbf{k}_{4}\right) \mathcal{R}_{k_{1}} \mathcal{R}_{k_{2}} \mathcal{R}_{k_{3}} \mathcal{R}_{k_{4}}\right], \\
& \quad \equiv\left(\frac{1}{c_{s}^{2}}-1\right) \frac{P_{\zeta, 0}}{32(2 \pi)\left|c_{s}\right|} \int\left(\prod_{i=1}^{4} d^{3} k_{i}\right) \delta^{(3)}\left(\sum_{i=1}^{4} k_{i}\right) \times \sum_{i=1}^{3} \tilde{H}_{4, i} .
\end{aligned}
$$

\section{Trispectrum: general formulas}

In this section, I gather different results obtained in the trispectrum computation which although useful their long form is not very illuminating.

\footnotetext{
${ }^{9}$ Note that there is a factor of $a$ different from [34] because here the Hamiltonian is in conformal time.
} 


\section{C.1 Contact interaction: contributions from $\mathcal{I}_{3}$}

The first expression is the result of the integral in eq. (3.35)

$$
\begin{aligned}
& \mathcal{I}_{3}\left(k_{1}, k_{2}, k_{3}, k_{4}\right)=\frac{\left|c_{s}\right|}{k_{1}^{4}}\left(\mathbf{k}_{1} \cdot \mathbf{k}_{2}\right)\left(\mathbf{k}_{3} \cdot \mathbf{k}_{4}\right) \times \\
& \quad\left[\left[-k_{1}^{3} \mathcal{F}\left[0, p_{1}\right]-k_{1}^{3} p_{1} \mathcal{F}\left[1, p_{1}\right]+\left(k_{1}\left(k_{2}+k_{3}+k_{4}\right)-k_{3} k_{4}-k_{2}\left(k_{3}+k_{4}\right)\right) k_{1} \mathcal{F}\left[2, p_{1}\right]+\right.\right. \\
& \left.\quad+\left(-k_{2} k_{3} k_{4}+k_{1}\left(k_{3} k_{4}+k_{2}\left(k_{3}+k_{4}\right)\right)\right) \mathcal{F}\left[3, p_{1}\right]+k_{2} k_{3} k_{4} \mathcal{F}\left[4, p_{1}\right]\right]+3 \text { perm. }+ \\
& \quad+k_{1}^{3} \mathcal{F}\left[0, q_{t}\right]+k_{1}^{3} q_{t} \mathcal{F}\left[1, q_{t}\right]+\left(k_{3} k_{4}+k_{2}\left(k_{3}+k_{4}\right)+k_{1}\left(k_{2}+k_{3}+k_{4}\right)\right) k_{1} \mathcal{F}\left[2, q_{t}\right]+ \\
& \left.\quad+\left(k_{2} k_{3} k_{4}+k_{1}\left(k_{3} k_{4}+k_{2}\left(k_{3}+k_{4}\right)\right)\right) \mathcal{F}\left[3, q_{t}\right]+k_{2} k_{3} k_{4} \mathcal{F}\left[4, q_{t}\right]\right]+5 \text { perm. }
\end{aligned}
$$

For shapes far from the flattened limit and for $\alpha \gg 1$ it becomes

$$
\begin{aligned}
& \mathcal{I}_{3}^{\text {non-flat }}\left(k_{1}, k_{2}, k_{3}, k_{4}\right)=\left|c_{s}\right| \frac{\left(\mathbf{k}_{1} \cdot \mathbf{k}_{2}\right)\left(\mathbf{k}_{3} \cdot \mathbf{k}_{4}\right)}{k_{1}^{4}} \times \\
& \quad\left[\left[\frac { 1 } { p _ { 1 } ^ { 5 } } \left(k_{1}^{3}\left(p_{1}-1\right) p_{1}^{3}+6 k_{2} k_{3} k_{4}\left(p_{1}-4\right)+\left(k_{2}+k_{3}+k_{4}\right)\left(p_{1}-2\right) p_{1}^{2} k_{1}^{2}+\right.\right.\right. \\
& \left.\left.\quad+2\left(k_{3} k_{4}+k_{2}\left(k_{3}+k_{4}\right)\right) k_{1}\left(p_{1}-3\right) p_{1}\right)+3 \text { perm. }\right]- \\
& \quad-\frac{1}{q_{t}^{5}}\left(k_{1} q_{t}\left(k_{1} q_{t}\left(k_{1} q_{t}\left(q_{t}+1\right)+k_{4}\left(q_{t}+2\right)\right)+k_{3}\left(k_{1} q_{t}\left(q_{t}+2\right)+2 k_{4}\left(q_{t}+3\right)\right)\right)+\right. \\
& \left.\quad+k_{2}\left(k_{1} q_{t}\left(k_{1} q_{t}\left(q_{t}+2\right)+2 k_{4}\left(q_{t}+3\right)\right)+2 k_{3}\left(k_{1} q_{t}\left(q_{t}+3\right)+3 k_{4}\left(q_{t}+4\right)\right)\right)\right)+5 \text { perm. }
\end{aligned}
$$

\section{C.2 Exchanged diagram: equilateral limits}

In the equilateral limit, $k_{1}=k_{2}=k_{3}=k_{4}$, the different contributions to the trispectrum in the large- $\alpha$ limit are given by

$$
\mathcal{T}_{i}^{\text {equi }}=-\left(\frac{1}{\left|c_{s}^{2}\right|}+1\right)^{2} \frac{1}{k^{3} 2^{9}\left|c_{s}\right|^{2}} \mathcal{K}_{i}^{\text {equi }},
$$

where

$$
\begin{gathered}
\mathcal{K}_{1}^{\text {equi }}(k, k, k, k)=-4 \times 144\left(\frac{A}{c_{s}^{2}}\right)^{2} b_{12} k^{3}\left|c_{s}\right|^{6} \times \\
\left(\frac{4}{b_{12}^{6}}+\frac{4}{\left(b_{12}+2\right)^{6}}-\frac{8}{b_{12}^{3}\left(b_{12}+2\right)^{3}}-b_{12} \frac{93 b_{12}^{4}-1048 b_{12}^{2}+3600}{64\left(4-b_{12}^{2}\right)^{3}}\right)+\left(b_{12} \leftrightarrow\left\{b_{14}, b_{13}\right\}\right), \\
\mathcal{K}_{2}^{\text {equi }}(k, k, k, k)=\frac{3}{2}\left(\frac{A}{\left|c_{s}^{2}\right|}\right) \frac{k^{3}\left|c_{s}\right|^{4}}{\left(b_{12}-2\right)^{3} b_{12}^{5}\left(b_{12}+1\right)^{4}\left(b_{12}+2\right)^{6}} \\
\left(262144+1441792 b_{12}+2867200 b_{12}^{2}+1548288 b_{12}^{3}-2899968 b_{12}^{4}-4988928 b_{12}^{5}-1304064 b_{12}^{6}+\right. \\
2437632 b_{12}^{7}+1527552 b_{12}^{8}-494976 b_{12}^{9}-407520 b_{12}^{10}+573312 b_{12}^{11}+920192 b_{12}^{12}+543296 b_{12}^{13}+ \\
\left.43904 b_{12}^{14}-130766 b_{12}^{15}-67973 b_{12}^{16}-4424 b_{12}^{17}+211 b_{12}^{20}+2110 b_{12}^{19}+6394 b_{12}^{18}\right)+b_{12} \leftrightarrow\left\{b_{14}, b_{13}\right\}, \\
\mathcal{K}_{3}^{\text {equi }}(k, k, k, k)=-\frac{3}{2}\left(\frac{A}{\left|c_{s}^{2}\right|}\right) \frac{k^{3}\left|c_{s}\right|^{4}}{\left(b_{12}-2\right)^{3} b_{12}^{5}\left(b_{12}+1\right)^{5}\left(b_{12}+2\right)^{6}} \\
\left(-262144-1441792 b_{12}-2375680 b_{12}^{2}+565248 b_{12}^{3}+5849088 b_{12}^{4}+5480448 b_{12}^{5}-443904 b_{12}^{6}-\right. \\
3396096 b_{12}^{7}-1380096 b_{12}^{8}+782976 b_{12}^{9}+973344 b_{12}^{10}+585216 b_{12}^{11}+409408 b_{12}^{12}+218368 b_{12}^{13}+ \\
\left.77 b_{12}^{20}+770 b_{12}^{19}+2342 b_{12}^{18}-1528 b_{12}^{17}-24475 b_{12}^{16}-47218 b_{12}^{15}+19168 b_{12}^{14}\right)+b_{12} \leftrightarrow\left\{b_{14}, b_{13}\right\},
\end{gathered}
$$




$$
\begin{aligned}
& \mathcal{K}_{4}^{\text {equi }}(k, k, k, k)=\frac{16\left|c_{s}\right|^{2} k^{3}}{\left(b_{12}-2\right)^{3} b_{12}^{5}\left(b_{12}+1\right)^{5}\left(b_{12}+2\right)^{6}} \\
& \left(-524288-786432 b_{12}+1048576 b_{12}^{2}+2490368 b_{12}^{3}+196608 b_{12}^{4}-2555904 b_{12}^{5}-1449984 b_{12}^{6}+\right. \\
& 1046016 b_{12}^{7}+732416 b_{12}^{8}-767104 b_{12}^{9}-516800 b_{12}^{10}+469152 b_{12}^{11}+478064 b_{12}^{12}+51656 b_{12}^{13}- \\
& \left.435 b_{12}^{18}+3214 b_{12}^{17}+2984 b_{12}^{16}-32840 b_{12}^{15}+89780 b_{12}^{14}\right)+b_{12} \leftrightarrow\left\{b_{14}, b_{13}\right\} .
\end{aligned}
$$

For completeness I also give the integral form of $\mathcal{K}_{4}$ after inserting the mode functions in eq. $(3.45)$

$$
\begin{aligned}
\mathcal{K}_{4}\left(k_{1}, k_{2}, k_{3}, k_{4}\right)=-16\left|c_{s}\right|^{2} \int_{\alpha}^{0} d z_{1} \int_{\alpha}^{z_{1}} d z_{2}\left[( \mathbf { k } _ { 1 } \cdot \mathbf { k } _ { 2 } ) \left[\frac{k_{12}}{k_{4}^{5}}\left(\mathbf{k}_{3} \cdot \mathbf{k}_{4}\right) e^{p_{2} z_{1}+p_{1} z_{2}} \times\right.\right. \\
\left(k_{4}\left(z_{1}\left(-e^{\frac{2 k_{3} z_{1}}{k_{4}}}+e^{2 z_{1}}+2\right)-e^{\frac{2 k_{3} z_{1}}{k_{4}}}-e^{2 z_{1}}+2\right)+\right. \\
\left.k_{3} z_{1}\left(z_{1}\left(e^{\frac{2 k_{3} z_{1}}{k_{4}}}+e^{2 z_{1}}+2\right)+e^{\frac{2 k_{3} z_{1}}{k_{4}}}-e^{2 z_{1}}+2\right)\right) \times \\
\left(k _ { 4 } z _ { 2 } \left(k_{1}\left(e^{\frac{2 k_{12} z_{1}}{k_{4}}}+e^{\frac{2 k_{1} z_{2}}{k_{4}}}-e^{\frac{2 k_{2} z_{2}}{k_{4}}}-e^{\frac{2 k_{12} z_{2}}{k_{4}}}+2\right)+\right.\right. \\
\left.k_{2}\left(e^{\frac{2 k_{12} z_{1}}{k_{4}}}-e^{\frac{2 k_{1} z_{2}}{k_{4}}}+e^{\frac{2 k_{2} z_{2}}{k_{4}}}-e^{\frac{2 k_{12} z_{2}}{k_{4}}}+2\right)\right)+ \\
+k_{1} k_{2} z_{2}^{2}\left(e^{\frac{2 k_{12} z_{1}}{k_{4}}}+e^{\frac{2 k_{1} z_{2}}{k_{4}}}+e^{\frac{2 k_{2} z_{2}}{k_{4}}}-e^{\frac{2 k_{12} z_{2}}{k_{4}}}+2\right)+ \\
\left.k_{4}^{2}\left(e^{\frac{2 k_{12} z_{1}}{k_{4}}}-e^{\frac{2 k_{1} z_{2}}{k_{4}}}-e^{\frac{2 k_{2} z_{2}}{k_{4}}}-e^{\frac{2 k_{12} z_{2}}{k_{4}}}+2\right)\right)+ \\
2 \text { perm.] }+2 \text { perm. }]+5 \text { perm. }
\end{aligned}
$$

\section{References}

[1] K. Freese, J. A. Frieman and A. V. Olinto, Natural inflation with pseudo - Nambu-Goldstone bosons, Phys. Rev. Lett. 65 (1990) 3233-3236.

[2] E. Silverstein and A. Westphal, Monodromy in the CMB: Gravity Waves and String Inflation, Phys. Rev. D78 (2008) 106003, [0803.3085].

[3] N. Kaloper and L. Sorbo, A Natural Framework for Chaotic Inflation, Phys. Rev. Lett. 102 (2009) 121301, [0811.1989].

[4] Planck collaboration, Y. Akrami et al., Planck 2018 results. X. Constraints on inflation, 1807.06211.

[5] A. Hebecker and P. Henkenjohann, Gauge and gravitational instantons: From 3-forms and fermions to Weak Gravity and flat axion potentials, JHEP 09 (2019) 038, [1906.07728].

[6] S. Cremonini, Z. Lalak and K. Turzynski, Strongly Coupled Perturbations in Two-Field Inflationary Models, JCAP 1103 (2011) 016, [1010.3021].

[7] A. Achucarro, J.-O. Gong, S. Hardeman, G. A. Palma and S. P. Patil, Mass hierarchies and non-decoupling in multi-scalar field dynamics, Phys. Rev. D84 (2011) 043502, [1005.3848].

[8] A. Achucarro, V. Atal, S. Cespedes, J.-O. Gong, G. A. Palma and S. P. Patil, Heavy fields, reduced speeds of sound and decoupling during inflation, Phys. Rev. D86 (2012) 121301, [1205.0710]. 
[9] S. Cespedes, V. Atal and G. A. Palma, On the importance of heavy fields during inflation, JCAP 1205 (2012) 008, [1201.4848].

[10] S. Renaux-Petel and K. Turzyński, Geometrical Destabilization of Inflation, Phys. Rev. Lett. 117 (2016) 141301, [1510.01281].

[11] A. R. Brown, Hyperbolic Inflation, Phys. Rev. Lett. 121 (2018) 251601, [1705. 03023].

[12] S. Mizuno and S. Mukohyama, Primordial perturbations from inflation with a hyperbolic field-space, Phys. Rev. D96 (2017) 103533, [1707.05125].

[13] P. Christodoulidis, D. Roest and E. I. Sfakianakis, Angular inflation in multi-field $\alpha$-attractors, JCAP 1911 (2019) 002, [1803.09841].

[14] S. Garcia-Saenz, S. Renaux-Petel and J. Ronayne, Primordial fluctuations and non-Gaussianities in sidetracked inflation, JCAP 1807 (2018) 057, [1804.11279].

[15] R. Bravo, G. A. Palma and S. Riquelme, A Tip for Landscape Riders: Multi-Field Inflation Can Fulfill the Swampland Distance Conjecture, JCAP 2002 (2020) 004, [1906.05772].

[16] V. Aragam, S. Paban and R. Rosati, Multi-field Inflation in High-Slope Potentials, 1905.07495.

[17] S. Garcia-Saenz, L. Pinol and S. Renaux-Petel, Revisiting non-Gaussianity in multifield inflation with curved field space, JHEP 01 (2020) 073, [1907.10403].

[18] T. Bjorkmo and M. C. D. Marsh, Hyperinflation generalised: from its attractor mechanism to its tension with the 'swampland conditions', JHEP 04 (2019) 172, [1901.08603].

[19] T. Bjorkmo, Rapid-Turn Inflationary Attractors, Phys. Rev. Lett. 122 (2019) 251301, [1902.10529].

[20] D. Chakraborty, R. Chiovoloni, O. Loaiza-Brito, G. Niz and I. Zavala, Fat Inflatons, Large Turns and the $\eta$-problem, JCAP 2001 (2020) 020, [1908.09797].

[21] P. Christodoulidis, D. Roest and E. I. Sfakianakis, Scaling attractors in multi-field inflation, JCAP 1912 (2019) 059, [1903.06116].

[22] D. Langlois and S. Renaux-Petel, Perturbations in generalized multi-field inflation, JCAP 0804 (2008) 017, [0801.1085].

[23] C. Cheung, P. Creminelli, A. L. Fitzpatrick, J. Kaplan and L. Senatore, The Effective Field Theory of Inflation, JHEP 03 (2008) 014, [0709. 0293].

[24] A. Achucarro, J.-O. Gong, S. Hardeman, G. A. Palma and S. P. Patil, Features of heavy physics in the CMB power spectrum, JCAP 1101 (2011) 030, [1010.3693].

[25] A. Achucarro, J.-O. Gong, S. Hardeman, G. A. Palma and S. P. Patil, Effective theories of single field inflation when heavy fields matter, JHEP 05 (2012) 066, [1201.6342].

[26] M. M. Anber and L. Sorbo, Naturally inflating on steep potentials through electromagnetic dissipation, Phys. Rev. D81 (2010) 043534, [0908.4089].

[27] N. Barnaby, R. Namba and M. Peloso, Phenomenology of a pseudo-scalar inflaton: Naturally large nongaussianity, JCAP 1104 (2011) 009, [1102.4333].

[28] R. Z. Ferreira and M. S. Sloth, Universal constraints on axions from inflation, JHEP 12 (2014) 139, [1409.5799].

[29] R. Z. Ferreira and A. Notari, Thermalized axion inflation, JCAP 1709 (2017) 007, [1706.00373].

[30] S. Garcia-Saenz and S. Renaux-Petel, Flattened non-Gaussianities from the effective field theory of inflation with imaginary speed of sound, JCAP 1811 (2018) 005, [1805.12563].

[31] T. Bjorkmo, R. Z. Ferreira and M. C. D. Marsh, Mild Non-Gaussianities under Perturbative Control from Rapid-Turn Inflation Models, JCAP 1912 (2019) 036, [1908.11316]. 
[32] J. Fumagalli, S. Garcia-Saenz, L. Pinol, S. Renaux-Petel and J. Ronayne, Hyper-Non-Gaussianities in Inflation with Strongly Nongeodesic Motion, Phys. Rev. Lett. 123 (2019) 201302, [1902.03221].

[33] X. Chen, M.-x. Huang, S. Kachru and G. Shiu, Observational signatures and non-Gaussianities of general single field inflation, JCAP 0701 (2007) 002, [hep-th/0605045].

[34] X. Chen, B. Hu, M.-x. Huang, G. Shiu and Y. Wang, Large Primordial Trispectra in General Single Field Inflation, JCAP 0908 (2009) 008, [0905.3494].

[35] E. Calzetta and B. L. Hu, Closed-time-path functional formalism in curved spacetime: Application to cosmological back-reaction problems, Physical Review D 35 (jan, 1987) 495-509.

[36] S. Weinberg, Quantum contributions to cosmological correlations, hep-th/0506236v1.

[37] R. Z. Ferreira, J. Ganc, J. Noreña and M. S. Sloth, On the validity of the perturbative description of axions during inflation, JCAP 1604 (2016) 039, [1512.06116].

[38] C. Germani and I. Musco, Abundance of Primordial Black Holes Depends on the Shape of the Inflationary Power Spectrum, Phys. Rev. Lett. 122 (2019) 141302, [1805.04087].

[39] P. D. Meerburg et al., Primordial Non-Gaussianity, 1903.04409.

[40] Planck collaboration, Y. Akrami et al., Planck 2018 results. IX. Constraints on primordial non-Gaussianity, 1905.05697.

[41] L. Senatore, K. M. Smith and M. Zaldarriaga, Non-Gaussianities in Single Field Inflation and their Optimal Limits from the WMAP 5-year Data, JCAP 1001 (2010) 028, [0905.3746].

[42] P. D. Meerburg, J. P. van der Schaar and M. G. Jackson, Bispectrum signatures of a modified vacuum in single field inflation with a small speed of sound, JCAP 1002 (2010) 001, [0910.4986].

[43] D. Seery and J. E. Lidsey, Primordial non-Gaussianities in single field inflation, JCAP 0506 (2005) 003, [astro-ph/0503692]. 